\title{
LINC00886, as a Biomarker Indicative of Thyroid Cancer Dedifferentiation, Negatively Regulates the Malignancy in Anaplastic Thyroid Cancer
}

Ben Ma

Shanghai Stomatological Disease Center

Yi Luo

Fudan University Shanghai Cancer Center

Weibo Xu

Fudan University Shanghai Cancer Center

Litao Han

Fudan University Shanghai Cancer Center

Wanlin Liu

Fudan University Shanghai Cancer Center

Hongyi Jiang

Fudan University Shanghai Cancer Center

Xiao Wang

Fudan University Shanghai Cancer Center

Shishuai Wen

Fudan University Shanghai Cancer Center

Tian Liao

Fudan University Shanghai Cancer Center

Wenjun Wei

Fudan University Shanghai Cancer Center

Qinghai Ji

Fudan University Shanghai Cancer Center

Yu Wang ( $\square$ neck130@hotmail.com )

Fudan University Shanghai Cancer Center

\section{Research}

Keywords: ATC, LINC00886, PKR, elF2a

Posted Date: July 21st, 2020

DOI: https://doi.org/10.21203/rs.3.rs-42533/v1 
License: (c) (i) This work is licensed under a Creative Commons Attribution 4.0 International License. Read Full License 


\section{Abstract}

Backgroud: Anaplastic thyroid cancer (ATC) is the most aggressive type of thyroid cancer. This study aimed to identify a long non-cdoing RNA (IncRNA) as a specific biomarker associated with ATC, and further investigated its biological function and molecular mechanism underlying regulation of malignancy in ATC.

Methods: We searched for IncRNAs associated with dedifferentiation and screened out specific IncRNAs significantly deregulated in ATC by using transcriptome data of dedifferentiation cancers from Fudan University Shanghai Cancer Center (FUSCC) and the Gene Expression Omnibus (GEO) database. The above IncRNAs were analyzed to identify a useful biomarker in thyroid cancer patients from the FUSCC, GEO and The Cancer Genome Atlas, which was further investigated for its functional roles and molecular mechanism in ATC in vitro.

Results: The clinicopathological association analyses revealed that LINC00886 was correlated with thyroid cancer dedifferentiation and significantly suppressed in ATC. In vitro, LINC00886 was confirmed to negatively regulate cell proliferation, colony formation and cell migration and invasion of ATC. LINC00886 physically interacted with protein kinase $R(P K R)$ and affected its stability through ubiquitin/proteasome-dependent degradation pathway in the ATC cell. Decreased PKR caused by LINC00886 downregulation, enhanced the activity of eukaryotic initiation factor $2 a$ (elF2a) via reducing the phosphorylation of elF $2 a$ and thus promoted protein synthesis to maintain the ATC malignancy.

Conclusions: Our findings identify LINC00886 as a novel biomarker of thyroid cancer and suggest that the LINC00886/PKR/elF2a signaling axis is a potential therapeutic target in ATC.

\section{Background}

Of thyroid cancer, anaplastic thyroid cancer (ATC) is a rare but extremely refractory form of malignancy. Although the incidence of ATC is $1 \%-2 \%$ [1], the mortality rate varies from $19.9-39 \%$ in all thyroid cancer types according to previous studies [2, 3]. A series of studies support a stepwise tumoral progression from well-differentiated cancer to poorly differentiated thyroid cancer (PDTC) and finally to ATC through accumulation of key genetic alterations [4-7], but the molecular mechanism remains unclarified concerning malignant formation of ATC.

High-throughput RNA sequencing uncovers universal existence of a number of tumor-specific long noncoding RNAs (IncRNAs) in cancer [8-10]. LncRNAs are defined as transcripts in length > $200 \mathrm{nt}$ with no protein coding potential, which play critical biological roles in cellular processes by regulating chromatin structure $[11,12]$, scaffolding or sequestering protein and mRNA to exert effects on their stability, localization and activity [13-15], and acting as miRNA sponges. LncRNAs have been widely reported to mediate regulation of cell proliferation, cell cycle, apoptosis and cell invasion and migration in cancer [9, $12,18]$, of which some are identified as biological markers indicating disease status and prognosis on one aspect $[19,20]$, and some may become potential therapeutic targets on the other aspect [21]. 
The present study aimed to identify a IncRNA that was specifically deregulated in ATC, and further to investigate its biological function and molecular mechanism underlying regulation of malignancy in ATC. We used transcriptome data of dedifferentiation thyroid cancer (DDTC) cases from Fudan University Shanghai Cancer Center (FUSCC) and the Gene Expression Omnibus (GEO) database to screen out and validate differential IncRNAs in ATC compared with normal thyroid (NT) and papillary thyroid cancer (PTC). LINC00886, as a suppressor biomarker in ATC, was identified in our clinicopathological association analyses, and the further experimental study indicated LINC00886 negatively regulated the malignancy of ATC via the protein kinase R (PKR)/eukaryotic initiation factor $2 a$ (elF2a) signaling axis.

\section{Materials And Methods}

\section{RNA sequencing data from FUSCC}

High-throughput RNA sequencing was performed in six NT tissues, five PTC tissues and five DDTC tissues from PTC cases undergoing dedifferentiation, which were described in our previous study [22]. A total of fifteen samples were included in the present study because of the bias of the RNA sequencing data from a NT sample. As shown in Fig. S1A, the transcriptome difference among DDTCs, PTCs and NTs was significant via the correlation matrix analyses for the RNA sequencing data of the enrolled fifteen samples. Moreover, nine ATC, five PDTC, sixteen PTC and twenty-one NT samples from FUSCC were used to validate expression of the IncRNAs screened out by RNA sequencing.

\section{Transcriptional profiles from the GEO database and The Cancer Genome Atlas (TCGA) database}

As described in our previous study [22], we obtained raw microarray cell intensity files of thyroid cancer including GSE29265, GSE33630 [23, 24], GSE53157 [25], GSE65144 [26] and GSE76039 [5] from the GEO database (http://www.ncbi.nlm.nih.gov/geo/) [27, 28]. The same chip platform (Affymetrix Human Genome U133 Plus 2.0 Array) was utilized by the files of the GEO cohort. The files were backgroundadjusted and normalized using Robust Multichip Average [29], and the ComBat method was used to remove the batch effects. All probes were mapped to the latest version of the NetAffx Annotation File. If a gene symbol was recorded with multiple probes, the average value was used as its expression level. The combined GEO cohort included the GSE29265 cohort (20 NTs, 20 PTCs and 9 ATCs), the GSE33630 cohort (45 NTs, 49 PTCs and 11 ATCs), the GSE53157 cohort (3 NTs, 15 PTCs and 5 PDTCs), the GSE65144 cohort (13 NTs and 12 ATCs) and the GSE76039 cohort (17 PDTCs and 20 ATCs). TCGA cohort data were available at the website of DATA SETS of University of California Santa Cruz (UCSC) Xena (THCA_22 datasets: https://xenabrowser.net/datapages/; https://www. biorxiv.org/content/early/2018/08/28/326470) and The cBioPortal for Cancer Genomics (http://www.cbioportal.org/). The data from the TCGA cohort and the GEO cohort were described in detail in our previous study [22].

\section{Cell culture and reagents}


CAL-62 and B-CPAP cell lines were purchased from the Shanghai Cell Bank Type Culture Collection Committee (CBTCCC, Shanghai, China). We cultured the cells at $37^{\circ} \mathrm{C}$ in a humidified incubator with $5 \%$ $\mathrm{CO}_{2}$. CAL-62 and B-CPAP were cultured in Dulbecco's modified Eagle's medium (DMEM) (Invitrogen, Carlsbad, CA, USA) and Roswell Park Memorial Institute (RPMI) 1640 medium (Invitrogen), respectively, supplemented with $10 \%$ foetal bovine serum (FBS) (HyClone, Logan, UT, USA), $100 \mathrm{U} / \mathrm{ml}$ penicillin and $100 \mu \mathrm{g} / \mathrm{ml}$ streptomycin (Invitrogen), and $8 \mathrm{mg} / \mathrm{l}$ antibiotic tylosin tartrate against mycoplasma (SigmaAldrich, St. Louis, Missouri, USA). The proteasome inhibitor MG132 and Cycloheximide (CHX) were purchased from Sigma-Aldrich.

\section{Protein-coding potential of LINC00886}

The Coding Potential Assessment Tool (CPAT) (http://lilab.research.bcm.edu/cpat/) and Coding Potential Calculator (CPC) (http://cpc.cbi.pku.edu.cn/) were used to predict the protein-coding potential of LINC00886. ACTB and GAPDH were used as coding RNA controls, and NEAT1 and MALAT1 were used as non-coding RNA controls.

\section{$5^{\prime}$ and $3^{\prime}$ rapid amplification of the cDNA ends (RACE)}

5'and 3'RACE were used to determine the initiation and termination sites of the LINC00886 transcript with a SMARTer RACE cDNA Amplification kit (Clontech, California, USA), according to manufacturer's instructions. The sequences for the gene-specific PCR primers used for 5'and 3'RACE analysis are listed in Table S1.

\section{Northern blot}

LINC00886 was detected using a NorthernMax Kit from Ambion (Thermo Fisher Scientific, Carlsbad, California, USA) and DIG Northern starter Kit (Roche, Indianapolis, Indiana, USA) with Digoxin-labelled RNA probes in the CAL-62 cells. The primers of the LINC00886's probes are listed in Table S1.

\section{Subcellular distribution}

We extracted cytoplasmic and nuclear fractions of the CAL-62 cells using the Nuclear/Cytoplasmic Isolation kit (Thermo Fisher Scientific, Carlsbad, California, USA). $\beta$-actin served as the cytoplasmic endogenous control. U2 served as the nuclear endogenous control.

\section{Overexpression and knockdown of LINC00886 and PKR}

The human LINC00886 sequence was cloned from CAL-62 cell CDNA and cloned into the expression

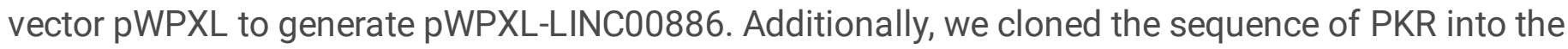
pCMV-N-Flag vector. The CAL-62 cells were transfected with pWPXL-LINC00886 and pCMV-PKR-N-Flag using Lipofectamine 2000 (Invitrogen) according to the manufacturer's instructions. We used Ribo ${ }^{\text {TM }}$ IncRNA Smart Silencer for LINC00886 knockdown, and small interfering RNA (siRNA) oligonucleotides targeting PKR was used for PKR knockdown. The IncRNA smart silencer is a mixture of three anti-sense oligonucleotides (ASO) and three siRNAs. The sequences of IncRNA smart silencer against LINC00886, siRNAs targeting PKR and the negative control (NC) siRNA were provided in Table S1, which were 
purchased from RiboBio (RiboBio Biotechnology, Guangzhou, China) and Genomeditech (Genomeditech, Shanghai, China). Transfections with IncRNA smart silencer and siRNA were performed with Lipofectamine RNAiMAX (Invitrogen).

\section{Reverse transcription PCR and quantitative real-time PCR (qPCR)}

TRIzol reagent (Invitrogen) was used to extract total RNA from the tissue specimens and cell lines. Firststrand cDNA was synthesized using the PrimeScript ${ }^{\text {TM }}$ Reverse Transcriptase kit (Takara, Dalian, China). We utilized quantitative qPCR to detect relative RNA levels on a 7900 Real-Time PCR System with the SDS 2.3 software sequence detection system (Applied Biosystems, USA) using the SYBR Green (Takara) method. The sequences for the gene-specific primers are listed in Table S1. $\beta$-actin is an internal control to quantify LINC00886 and the mRNA levels of other genes. The relative levels of RNA are calculated by the comparative CT $\left(2^{-\triangle \Delta C T}\right)$.

\section{Cell proliferation assay and colony formation assay}

The CAL-62 cells were seeded in 96-well flat-bottomed plates, with each well containing 2000 cells in $100 \mu \mathrm{l}$ of cell suspension. Cell viability was measured using Cell Counting Kit-8 (CCK-8) assays (YEASEN, Shanghai, China). We repeated each assay with five replicates for three times and measured continuously for 5 days. Cells were seeded in 6-well culture dishes, and after 1 week- 2 weeks, cell colonies were fixed with methanol, stained with crystal violet (Sigma-Aldrich, St. Louis, MO), and counted based on the colony formation number.

\section{Cell invasion assay and migration assay}

For the migration assays, the $5 \times 10^{4} \mathrm{CAL}-62$ cells were added to the Millicell chambers with $100 \mu \mathrm{l}$ of serum-free medium, which were ahead put inside the 24-well culture dishes containing $500 \mu$ of DMEM with $10 \%$ FBS. Invasion assays were performed similarly with coating the filters with Matrigel (BD Biosciences, Franklin Lakes, New Jersey, USA). The cell invasion and migration assays were performed in triplicate. After $24 \mathrm{~h}$ at $37{ }^{\circ} \mathrm{C}$ in an incubator with $5 \% \mathrm{CO}_{2}$, migrated cells were fixed with methanol and stained with crystal violet, and cell numbers were counted in five random fields.

\section{RNA sequencing}

We extracted total RNA from the CAL-62 cells transfected with LINC00886 smart silencer and NC-siRNA using TRIzol reagent. The RNA samples from si-LINC00886 and NC cells were used for RNA sequencing as mentioned in our previous study [22]. The RNA sequencing data were exhibited in Table S2.

\section{Gene Set Enrichment Analysis (GSEA)}

GSEA was performed based on the RNA sequencing data of si-LINC00886 and NC CAL-62 cells and transcriptional profiles of the GEO ATCs and the TCGA PTCs using GSEA software, Version 2.0, which was obtained from the Broad Institute (http://www.broad.mit.edu/gsea), as previously described. Enrichment Map was used for visualization of the GSEA results. Normalized enrichment score (NES) and 
false discovery rate (FDR) were used to sort the pathways enriched in each phenotype after gene set permutations were performed 1000 times for each analysis.

\section{Western blot}

We used lysis buffer (YEASEN, Shanghai, China) with protease inhibitors (Roche, Indianapolis, IN, USA) to lyse CAL-62 cells. Protein concentrations were determined by the BCA method (Pierce, Therrmo Fisher Scientific Inc., Rockford, IL, USA). The protein samples were subjected to SDS/PAGE and transferred to PVDF membranes (Immobilon-P membrane, Millipore, Massachusetts, USA). The membranes were blocked with 5\% skimmed milk in TBS plus Tween 20 at room temperature for 1 hour, followed by incubation with target antibodies at $4{ }^{\circ} \mathrm{C}$ overnight. Information on the antibodies are provided in Table S3. After incubation with HRP-conjugated secondary antibodies for 1 hour, visualization of the protein bands was achieved by an enhanced chemiluminescent chromogenic substrate using the Enhanced Chemiluminescence Plus Western Blotting Detection System (GE Healthcare, Connecticut, USA) and LAS4000EPUV mini Luminescent Image Analyzer (GE Healthcare).

\section{RNA pull down}

Initially, LINC00886 and antisense-LINC00886 RNAs were labelled by the Biotin RNA Labeling Mix (Roche, USA) and purified with an RNeasy Mini Kit (QIAGEN, USA). Then, the biotinylated RNAs were incubated with streptavidin beads (Invitrogen, USA), and the streptavidin beads combined with and without RNAs were mixed with protein extracts of CAL- 62 cells at $4{ }^{\circ} \mathrm{C}$ on a rotator overnight. The beads were washed gently three times in $1 \times$ washing buffer ( $5 \mathrm{mM}$ Tris- $\mathrm{HCl}, 1 \mathrm{M} \mathrm{NaCl}, 0.5 \mathrm{mM}$ EDTA, and 0.005\% Tween 20), followed by mix with DEPC and $5 \times$ SDS buffer. The proteins were separated by gel electrophoresis and visualized by silver staining. Specific bands were identified by mass spectrometry analysis and retrieved in the human RefSeq protein database (National Center for Biotechnology Information), using Mascot version 2.4.01 (Matrix Science, London, UK). The retrieved protein was detected by western blot. The primers of LINC00886 are provided in Table S1.

\section{RNA immunoprecipitation (RIP)}

We performed RIP assays using the Magna RIP RNA-binding protein immunoprecipitation kit (Millipore, Massachusetts, USA) according to the manufacturer's instructions. The CAL-62 cells were lysed in RIP lysis buffer, and the lysate products were immunoprecipitated with PKR or IgG antibody combined with protein magnetic beads. Next, we immobilized the magnetic beads bound complexes and washed unbound materials. The RNAs were extracted using the Total RNA isolation kit (Thermo Fisher Scientific Inc) and analyzed by qPCR and real-time PCR. For the RIP assays of deletion mutants, plasmids with FLAG-full-length and truncated PKR were transiently transfected into the CAL-62 cells, and the cell lysates were immunoprecipitated with the indicated antibodies.

\section{Immunoprecipitation}

Immunoprecipitation assays were performed using the Pierce ${ }^{\text {TM }}$ Classic Magnetic IP/Co-IP Kit (Thermo Fisher Scientific Inc). The CAL-62 cells were transfected with the indicated plasmids and then lysed in ice- 
cold IP Lysis/Wash Buffer (pH 7.4, 0.025M Tris, 0.15M NaCl, 0.001M EDTA, 1\% NP40, 5\% glycerol). Cell lysate were incubated with anti-PKR antibody for 1-2 hours at room temperature or overnight at $4{ }^{\circ} \mathrm{C}$. And then, the antigen/antibody complexes were mixed with protein magnetic beads (Thermo Fisher Scientific Inc.) at room temperature for one hour. The beads were washed twice with IP Lysis/Wash Buffer and once with purified water, and the antigen/antibody complexes were eluted. The immuno-complexes were analysed by SDS/PAGE and immunoblotting with anti-PKR, anti-ubiquitin and anti- $\beta$-actin antibody.

\section{Statistical Analysis}

Continuous variables were respectively expressed as the mean \pm standard error of the mean (SEM) from at least three independent experiments and mean \pm standard deviation (SD) in analyses of associations between clinical parameters and molecular variables, and categorical data were summarized with frequencies and percentages. Student's t-test and one way analysis of variance were performed to evaluate the differences of continuous variables between two groups and among more than two groups, respectively. Associations between continuous variables and categorical variables were evaluated using Mann-Whitney $U$ tests for two groups and Kruskal-Wallis tests for more than two groups. $\chi 2$ and Fisher's exact test were used for categorical variables. Based on the median value of thyroid differentiation score (TDS), we divided the PTC patients from the TCGA cohort into a low-differentiated group and a highdifferentiated group. Pearson's correlation was performed to analyze the correlation of LINC00886 with the TDS. Nonparametric receiver operating characteristic (ROC) analyses were performed to calculate the area under curve (AUC) for LINC00886 that would be predictive of dedifferentiation. Univariate logistic regression analyses were performed to determine associations of LINC00886 with clinicopathological factors in PTC patients from TCGA. Furthermore, LINC00886 was tested for overall survival (OS) analyses in thyroid cancer patients using the GSE76039 cohort [5]. The Kaplan-Meier method was used to construct OS curves, and the univariate survival difference was determined by the log-rank test. A $p$ value $<0.05$ was considered significant. Data preparation and statistical analyses were performed using the SPSS for Windows (version 22.0; IBM Corp., Armonk, NY), the R software (version 3.5.1; R Foundation for Statistical Computing, Vienna, Austria) and the GraphPad Prism (version 6.01; GraphPad Software Inc., La Jolla, Calif).

\section{Results}

\section{LINC00886 is associated with thyroid cancer dedifferentiation and significantly suppressed in ATC}

We initially analyzed expression alteration of all IncRNAs based on high-through transcriptome data of five DDTCs, five PTCs and five NTs from FUSCC in order to screen out oncogenic IncRNAs associated with dedifferentiation of thyroid cancer. Of all IncRNAs, 65 IncRNAs (41 downregulated and 24 upregulated IncRNAs, $\log _{2}$ fold change $(F C) \geq 2, p<0.05$ ) were consistently found deregulated in DDTCs compared with both PTCs and NTs (Fig. 1A-1C and Table S4). Expression alterations of top ten IncRNAs were summarized (Fig. 1D and Table S5), of which LINC00886 was found to be significantly associated 
with thyroid cancer dedifferentiation among the FUSCC cohort, the GEO cohort and the TCGA cohort (Fig. S1B-S1J and Fig. 1E-1I). LINC00886 is located in 3q25.31 with the length of 3354 bp according to National Center for Biotechnology Information (NCBI) (https://www.ncbi.nlm.nih.gov/gene/? term=LINC00886) (Fig. S2A). The analyses of CPAT and CPC revealed LINC00886 is non-coding (Fig. S2B-S2C). The results of $5^{\prime}$ and 3' RACE (Fig. S2D) and northern blot analyses (Fig. S2E) confirmed LINC00886's full length and stable transcript in the ATC cell. Additionally, subcellular distribution analyses indicated LINC00886 existed in both cytoplasm and nucleus of B-CPAP and CAL-62, and LINC00886 dominantly distributed in the cytoplasm of CAL-62 (Fig. S2F).

In the FUSCC cohort, LINC00886 expression was confirmed to be downregulated in ATC compared with NT and PTC (Fig. 1E), as shown in the GEO cohort (Fig. 1F). Low LINC00886 expression significantly correlated with the decreased OS (Fig. 1G), the ATC phenotype, LNM, BRAFV600E mutation and the mutation in the PI3KCA/AKT/mTOR signaling pathway (Table 1 and Fig. S2G) in PDTC and ATC patients from the GSE76039 cohort. Moreover, suppressed LINC00886 expression was observed in low-TDS PTC in comparison with NT and high-TDS PTC in the TCGA cohort (Fig. 1H), and LINC00886 exhibited a positive correlation with TDS and a high prediction for the low-differentiated phenotype (Fig. $1 \mathrm{I}$ and Fig. S1I-S1J). LINC00886 was remarkably associated with aggressive behaviors and genetic mutations of PTC, such as high-risk histological subtypes, extrathyroidal extension (ETE), lymph node metastasis (LNM), T3/T4 stage and II-IV stage, BRAFV600E mutation, RAS mutation, EIF1AX mutation and BRAF-RAS score (Table 2, Fig. 1J and Fig. 2). 
Table 1

Clinicopathological and molecular correlations of LINC00886 expression in the GSE76039 cohort

\begin{tabular}{|c|c|c|c|c|}
\hline \multirow[t]{2}{*}{ Variables } & \multirow[t]{2}{*}{$N$} & \multicolumn{2}{|l|}{ LINC00886 } & \multirow[t]{2}{*}{$p$ value } \\
\hline & & High expression & Low expression & \\
\hline Age (years) Mean \pm SD & 37 & $64.76 \pm 14.8$ & $66.5 \pm 12.6$ & 0.709 \\
\hline Gender & & & & 1.000 \\
\hline Male & 3 & $2(66.7 \%)$ & $1(33.3 \%)$ & \\
\hline Female & 12 & $8(66.7 \%)$ & $4(33.3 \%)$ & \\
\hline NA & 12 & & & \\
\hline Histology & & & & 0.001 \\
\hline PDTC & 19 & $16(84.2 \%)$ & $3(15.8 \%)$ & \\
\hline ATC & 18 & $5(27.8 \%)$ & $13(72.2 \%)$ & \\
\hline T Stage & & & & 1.000 \\
\hline T1-T2 & 2 & $2(50.0 \%)$ & $0(50.0 \%)$ & \\
\hline T3-T4 & 27 & $14(51.9 \%)$ & $13(48.1 \%)$ & \\
\hline NA & 8 & & & \\
\hline LNM & & & & 0.046 \\
\hline NO & 10 & $8(80.0 \%)$ & $2(20.0 \%)$ & \\
\hline N1 & 17 & $6(35.3 \%)$ & $11(64.7 \%)$ & \\
\hline NA & 10 & & & \\
\hline Distant metastasis & & & & 0.179 \\
\hline MO & 6 & $5(83.3 \%)$ & $1(16.7 \%)$ & \\
\hline M1 & 17 & $8(47.1 \%)$ & $9(52.9 \%)$ & \\
\hline NA & 14 & & & \\
\hline Mutation count & 35 & $6.32 \pm 4.98$ & $9.75 \pm 9.11$ & 0.167 \\
\hline BRAF V600E & & & & 0.036 \\
\hline Mutation & 13 & $4(30.8 \%)$ & $9(69.2 \%)$ & \\
\hline
\end{tabular}

Notes: Italic and bold type indicates statistical significance

Abbreviations: SD, standard deviation; NA, not available; PDTC, poorly differentiated thyroid cancer; ATC, anaplastic thyroid cancer; LNM, lymph node metastasis. 


\begin{tabular}{|c|c|c|c|c|}
\hline \multirow[t]{2}{*}{ Variables } & \multirow[t]{2}{*}{$N$} & \multicolumn{2}{|l|}{ LINC00886 } & \multirow[t]{2}{*}{$p$ value } \\
\hline & & High expression & Low expression & \\
\hline Wild-type & 24 & 17 (70.8\%) & $9(29.2 \%)$ & \\
\hline RAS & & & & 0.491 \\
\hline Mutation & 12 & $8(66.7 \%)$ & $4(33.3 \%)$ & \\
\hline Wild-type & 25 & 13 (52.0\%) & $12(48.0 \%)$ & \\
\hline EIF1AX & & & & 1.000 \\
\hline Mutation & 5 & $3(60.0 \%)$ & $2(40.0 \%)$ & \\
\hline Wild-type & 32 & $18(56.3 \%)$ & $14(43.8 \%)$ & \\
\hline TERT promoter & & & & 0.185 \\
\hline Mutation & 20 & $9(45.0 \%)$ & $11(55.0 \%)$ & \\
\hline Wild-type & 17 & $12(70.6 \%)$ & $5(29.4 \%)$ & \\
\hline TP53 & & & & 0.196 \\
\hline Mutation & 16 & $7(43.8 \%)$ & $9(56.3 \%)$ & \\
\hline Wild-type & 21 & $14(66.7 \%)$ & $7(33.3 \%)$ & \\
\hline PI3KCA/AKT/mTOR & & & & 0.024 \\
\hline Mutation & 9 & $2(22.2 \%)$ & 7 (77.8\%) & \\
\hline Wild-type & 28 & 19 (67.9\%) & $9(32.1 \%)$ & \\
\hline Notes: Italic and bold & tes $\mathrm{s}$ & istical significance & & \\
\hline
\end{tabular}


Table 2

Clinicopathological and molecular correlations of LINC00886 expression in the TCGA cohort

\begin{tabular}{|c|c|c|c|c|}
\hline \multirow[t]{2}{*}{ Variables } & \multirow[t]{2}{*}{$N$} & \multicolumn{2}{|l|}{ LINC00886 } & \multirow[t]{2}{*}{$p$ value } \\
\hline & & High expression & Low expression & \\
\hline Age (years) Mean \pm SD & 492 & $47.2 \pm 15.9$ & $47.0 \pm 15.8$ & 0.375 \\
\hline Gender & & & & 0.761 \\
\hline Male & 133 & $68(51.1 \%)$ & $65(48.9 \%)$ & \\
\hline Female & 359 & $178(49.6 \%)$ & $181(50.4 \%)$ & \\
\hline Multifocality & & & & 0.532 \\
\hline Unifocal & 261 & $126(48.3 \%)$ & $135(51.7 \%)$ & \\
\hline Multifocal & 221 & $110(48.7 \%)$ & $116(51.3 \%)$ & \\
\hline Coexistent HT & & & & 0.470 \\
\hline Yes & 65 & $31(47.7 \%)$ & $34(52.3 \%)$ & \\
\hline No & 396 & $208(52.5 \%)$ & $188(47.5 \%)$ & \\
\hline Histological subtype & & & & $<0.001$ \\
\hline Low risk & 451 & $242(53.7 \%)$ & $209(46.3 \%)$ & \\
\hline High risk & 41 & $4(9.8 \%)$ & $37(90.2 \%)$ & \\
\hline T Stage & & & & 0.001 \\
\hline T1-T2 & 304 & $170(55.9 \%)$ & $134(44.1 \%)$ & \\
\hline T3-T4 & 185 & $76(41.1 \%)$ & $109(58.9 \%)$ & \\
\hline ETE & & & & $<0.001$ \\
\hline Yes & 148 & $53(35.8 \%)$ & $95(64.2 \%)$ & \\
\hline No & 328 & $183(55.8 \%)$ & $145(44.2 \%)$ & \\
\hline LNM & & & & $<0.001$ \\
\hline NO & 223 & $137(61.4 \%)$ & $86(38.6 \%)$ & \\
\hline N1 & 222 & $84(37.8 \%)$ & $138(62.2 \%)$ & \\
\hline
\end{tabular}

Notes: Italic and bold type indicates statistical significance

Abbreviations: TCGA, The Cancer Genomics Atlas; HT, Hashimoto's thyroiditis; Histological subtypes: low-risk subtypes including clasical variant, follicular variant, mixed papillary and follicular variant, encapsulated follicular variant and columnar cell variant, high-risk subtypes including tall-cell variant, diffuse sclerosing variant and cribriform morbular variant; ETE, extrathyroidal extension; LNM, lymph node metastasis; TNM stage, tumor-node-metastasis. 


\begin{tabular}{|c|c|c|c|c|}
\hline \multirow[t]{2}{*}{ Variables } & \multirow[t]{2}{*}{$N$} & \multicolumn{2}{|l|}{ LINC00886 } & \multirow[t]{2}{*}{$p$ value } \\
\hline & & High expression & Low expression & \\
\hline \multicolumn{4}{|l|}{ Distant metastasis } & 0.746 \\
\hline MO & 274 & $134(48.9 \%)$ & $140(51.1 \%)$ & \\
\hline M1 & 9 & $5(55.6 \%)$ & $4(44.4 \%)$ & \\
\hline \multicolumn{4}{|l|}{ TNM stage } & 0.034 \\
\hline I & 384 & $202(52.6 \%)$ & $182(47.4 \%)$ & \\
\hline IIIV & 105 & $43(41.0 \%)$ & $62(59.0 \%)$ & \\
\hline \multicolumn{4}{|l|}{ BRAF V600E } & $<0.001$ \\
\hline Mutation & 238 & $65(27.3 \%)$ & $173(72.7 \%)$ & \\
\hline Wild-type & 253 & $181(71.5 \%)$ & 72 (28.5\%) & \\
\hline \multicolumn{4}{|l|}{ TERT promoter } & 0.309 \\
\hline Mutation & 36 & $15(41.7 \%)$ & $21(58.3 \%)$ & \\
\hline Wild-type & 358 & $181(50.6 \%)$ & $177(49.4 \%)$ & \\
\hline \multicolumn{4}{|l|}{ TERT exon } & 1.000 \\
\hline Mutation & 2 & $1(50.0 \%)$ & $1(50.0 \%)$ & \\
\hline Wild-type & 489 & 245 (50.1\%) & $244(49.9 \%)$ & \\
\hline \multicolumn{4}{|l|}{ RAS } & $<0.001$ \\
\hline Mutation & 51 & $48(94.1 \%)$ & $3(5.9 \%)$ & \\
\hline Wild-type & 440 & $198(45.0 \%)$ & $242(55.0 \%)$ & \\
\hline \multicolumn{4}{|l|}{ EIF1AX } & 0.030 \\
\hline Mutation & 6 & $6(100.0 \%)$ & $0(0.0 \%)$ & \\
\hline Wild-type & 485 & $240(49.5 \%)$ & $245(50.5 \%)$ & \\
\hline \multicolumn{4}{|l|}{ ETV6_NTRK3 } & 0.061 \\
\hline Fusion & 5 & $5(100.0 \%)$ & $0(0.0 \%)$ & \\
\hline \multicolumn{5}{|c|}{ Notes: Italic and bold type indicates statistical significance } \\
\hline \multicolumn{5}{|c|}{$\begin{array}{l}\text { Abbreviations: TCGA, The Cancer Genomics Atlas; HT, Hashimoto's thyroiditis; Histological subtypes: } \\
\text { low-risk subtypes including clasical variant, follicular variant, mixed papillary and follicular variant, } \\
\text { encapsulated follicular variant and columnar cell variant, high-risk subtypes including tall-cell variant, } \\
\text { diffuse sclerosing variant and cribriform morbular variant; ETE, extrathyroidal extension; LNM, lymph } \\
\text { node metastasis; TNM stage, tumor-node-metastasis. }\end{array}$} \\
\hline
\end{tabular}




\begin{tabular}{|c|c|c|c|c|}
\hline \multirow[t]{2}{*}{ Variables } & \multirow[t]{2}{*}{$N$} & \multicolumn{2}{|l|}{ LINC00886 } & \multirow[t]{2}{*}{$p$ value } \\
\hline & & High expression & Low expression & \\
\hline Wild-type & 486 & $241(49.6 \%)$ & $245(50.4 \%)$ & \\
\hline CCDC6_RET & & & & 0.269 \\
\hline Fusion & 21 & $13(61.9 \%)$ & $8(38.1 \%)$ & \\
\hline Wild-type & 470 & $233(49.6 \%)$ & $237(50.4 \%)$ & \\
\hline PAX8_PPARG & & & & 0.123 \\
\hline Fusion & 4 & $4(100.0 \%)$ & $0(0.0 \%)$ & \\
\hline Wild-type & 487 & $242(49.7 \%)$ & $245(50.3 \%)$ & \\
\hline \multicolumn{5}{|c|}{ Notes: Italic and bold type indicates statistical significance } \\
\hline \multicolumn{5}{|c|}{$\begin{array}{l}\text { Abbreviations: TCGA, The Cancer Genomics Atlas; HT, Hashimoto's thyroiditis; Histological subtypes: } \\
\text { low-risk subtypes including clasical variant, follicular variant, mixed papillary and follicular variant, } \\
\text { encapsulated follicular variant and columnar cell variant, high-risk subtypes including tall-cell variant, } \\
\text { diffuse sclerosing variant and cribriform morbular variant; ETE, extrathyroidal extension; LNM, lymph } \\
\text { node metastasis; TNM stage, tumor-node-metastasis. }\end{array}$} \\
\hline
\end{tabular}

\section{LINC00886 negatively regulates ATC cell proliferation and cell migration and invasion}

The ATC cell, CAL-62, was used to validate the biological roles of LINC00886 in vitro. Knockdown of LINC00886 accelerated cell proliferation and colony formation of CAL-62 (Fig. 3A-3C), and LINC00886overexpressed CAL-62 by pWPXL-LINC00886 transfection showed significant inhibition of cell proliferation and colony formation (Fig. 3D-3F). Additionally, LINC00886 knockdown significantly increased the migration and invasion ability of CAL-62 (Fig. 3G), whereas LINC00886 overexpression decreased migration and invasion of CAL-62 (Fig. 3H). Furthermore, we performed GSEA using RNA sequencing data of CAL-62 cells of LINC00886 knockdown as well as transcriptome data of highLINC00886 and low-LINC00886 expression groups from the GEO ATC cohort and the TCGA PTC cohort. The biological signaling pathways were selected according to NES $>1.5$ and FDR $<0.05$ (Table S6), and overlapped analyses were conducted on the CAL-62 cell group, the GEO cohort and the TCGA cohort. As a result, two overlapped signaling pathways including epithelial-mesenchymal transition and mitotic spindle were found to be enriched in LINC00886-downregulation group (Fig. 3I-3J and Fig. S3).

Additionally, LINC00886 knockdown demonstrated to upregulate expression of $\beta$-catenin and vimentin by immunoblot, viceversa, LINC00886 overexpression reduce levels of both $\beta$-catenin and vimentin (Fig. 3K).

\section{LINC00886 targets PKR in the ATC cell}

RNA pull-down assays were performed to identify the proteins interacting with LINC00886 in ATC cells to investigate the molecular mechanism underlying how LINC00886 regulates the malignancy of ATC cell. 
The specific bands showed around $70 \mathrm{kd}$ in the sense LINC00886 group compared with the antisense group and the magnetic bead (MB) group in three independent LINC00886 pull-down experiments (Fig. 4A), which were then subjected to mass spectrometry. We identified eight potential LINC00886interacting proteins that may be involved in tumor activity according to unique peptide number $(>2)$ and peptide number (>2) (Table S7). Immunoblotting confirmed that the only one candidate, PKR, directly interacts with LINC00886 repeatedly (Fig. 4B and Fig. S2H). Furthermore, RIP assays demonstrated that the antibody of PKR enriched LINC00886, as determined by measuring co-precipitated RNA by qPCR and real-time PCR with agarose gel electrophoresis analysis (Fig. 4C-4D). The further RIP assays for truncated PKR showed that the domain (375-500 aa), including the elF2a binding site, physically correlates with LINC00886 (Fig. 4E). These results revealed that LINC00886 specifically interacted with PKR in the ATC cell.

\section{LINC00886 affects the PKR's stability in the ATC cell}

To investigate the impact of LINC00886 on PKR, we tested PKR expression at mRNA and protein levels in LINC00886-knockdown and LINC00886-overexpressed CAL-62 cells. It was found that knockdown or overexpression of LINC00886 exerted no effect on PKR mRNA levels (Fig. 5A-5B). However, LINC00886 knockdown significantly reduced the protein levels of PKR in CAL-62, whereas LINC00886 overexpression increased PKR protein levels (Fig. 5C). Additionally, after treatment of the protein synthesis inhibitor-CHX, the half-life of PKR protein significantly shortened in LINC00886-knockdown cell while overexpression of LINC00886 prolonged its half-life (Fig. 5D-5E). A proteasome inhibitor MG132 was used to treat LINC00886-knockdown and negative-control cells. On one aspect, endogenous PKR protein levels accumulated in the MG132-positive cell compared with MG132-negative cell. On the other aspect, the anti-degradation effect of MG132 on PKR protein attenuated with LINC00886 knockdown (Fig. 5F). Moreover, the ubiquitination levels of PKR increased significantly in the LINC00886-knockdown cell compared with the NC cell (Fig. 5G). The above results indicated that LINC00886 affected the PKR's stability by influencing its ubiquitin/proteasome-dependent degradation.

\section{The PKR/elF2a signaling axis mediates functional regulation of LINC00886}

To elucidate the biological roles of LINC00886-PKR interaction in ATC, we next analyzed the functions of PKR in CAL-62 in vitro. It was found that cell proliferation and colony formation were enhanced following knockdown of PKR by siRNA (Fig. 6A-6C), and PKR overexpression by pCMV-PKR-N-Flag transfection significantly inhibited cell proliferation and colony formation (Fig. 6D-6F). Moreover, the anti-proliferation effect of LINC00886 overexpression on CAL-62 was reversed by PKR knockdown (Fig. 6G), suggesting the suppression effect of LINC00886 on cell proliferation was PKR-dependent in ATC. It is well acknowledged that PKR acts as a tumor suppressor by phosphorylation of elF2a to inhibit protein synthesis, and then we verified the effects of LINC00886 on PKR/elF2a signaling by the immunoblotting assay. As shown in Fig. 6H, LINC00886 knockdown reduced the protein levels of total PKR and its activated formphosphoryted PKR (Thr 446), and loss of phospho-PKR (Thr 446) inhibited phosphorylation of elF2a (Ser 51). Contrariwise, LINC00886 overexpression accumulated total PKR, phospho-PKR (Thr 446) and 
phospho-elF2a (Ser 51). However, the total elF2a protein levels were not affected by both LINC00886 and PKR. Figure 6l exhibited the integrated mode of LINC00886/PKR/elF2a signaling axis in ATC.

\section{Discussion}

It is commonly observed that alteration of IncRNAs occurred in thyroid cancer [31]. A series of IncRNAs have been found to play oncogenic or suppressor roles in thyroid cancer, of which some are suggested as potential therapeutic targets [31-34]. To our knowledge, it is the first time that the differential IncRNAs have been screened out among NTs, PTCs and DDTCs from the FUSCC cohort to identify a IncRNA biomarker associated with dedifferentiation of thyroid cancer, which was specifically altered in ATC, and then was confirmed to regulate the malignancy of ATC.

In this study, we used the RNA sequencing data of the DDTC cases from FUSCC to screen out aberrant IncRNAs in DDTC, and further used the FUSCC cohort, the GEO cohort and the TCGA cohort to select a specific IncRNA indicative of thyroid cancer dedifferentiation, that was significantly deregulated in ATC. Consequently, we found that LINC00886 with the transcript length of 3354 bp located in 3q25.31 was significantly suppressed in ATC. Lan L et al reported that the significant decrease of LINC00886 was correlated with pathological grade in laryngeal squamous cell carcinoma [35]. Our study suggested that LINC00886 was a useful biomarker in thyroid cancer according to its clinicopathological associations. On one hand, LINC00886 showed a significant correlation with differentiation status of thyroid cancer. On the other hand, LINC00886 was associated with poor clinical outcomes of patients. Moreover, LINC00886 was confirmed to negatively regulate cell proliferation, cell colony formation, and cell migration and invasion in ATC in vitro, which was verified by enrichment of epithelial-mesenchymal transition and mitotic spindle signaling pathways after LINC00886 knockdown.

Interaction with proteins is typical of IncRNA's molecular mechanism in cancer cellular processes[36]. Our study identified PKR as the interacting partner of LINC00886 and demonstrated the negative regulatory roles of the LINC00886-PKR-elF2a signaling axis in ATC cell. LINC00886 binds to the active site of PKR, which contains the elF2 $a$ binding site. PKR, as an interferon-inducible double-stranded RNA protein kinase, is involved in various cellular pathways exerting effect on tumourigenesis and malignant progression. The dual roles of PKR have been uncovered in cancer biology. In general, PKR is considered to be a tumor suppressor, and the association of increased PKR expression with improved prognosis has been reported in many cancer types [37-40]. It is well-acknowledged that elF2a phosphorylation induced by PKR activation impairs the activity of elF2a and then inhibits protein synthesis, which is responsible for cell survival suppression [41, 42]. Furthermore, PKR has been suggested to play an essential role in the antitumor activity of tumour suppressors, such as p53 and PTEN $[43,44]$. By contrast, PKR is able to induce cancer cell survival through NF-KB activation, and it has been reported that PKR is overexpressed and associated with malignancy in several cancers $[43,45,46]$. In this study, we showed suppression effect of PKR/elF2 $a$ on ATC malignancy and found the regulatory mechanism for PKR via LINC00886. LINC00886 and PKR share highly similar functions in cell proliferation, supporting that PKR acts as a downstream target of LINC00886. 
Finally, some limitations have to be shown in this study. We did not analyzed LINC00886's prognostic value in ATC patients from the FUSCC due to lack of fresh ATC samples available for RNA extraction. The suppression effect of LINC00886 was not validated in transplant tumor of ATC cell in vivo, and we did not perform experiments concerning therapeutic inhibitors targeting the LINC00886-PKR-elF2a signaling axis. Therefore, in the next-step study, we aim to confirm the effect of LINC00886 as a biomarker in a large cohort of ATC patients from FUSCC. Meanwhile, it is necessary to confirm the effect of LINC00886 in a mouse model with transplant tumor of ATC cell, and to search for pharmaceutical inhibitors targeting the LINC00886-PKR-elF2a signaling axis.

\section{Conclusions}

In summary, we showed that LINC00886 is initially identified as a biomarker indicative of thyroid cancer dedifferentiation. LINC00886 is significantly suppressed in ATC, and inhibits ATC cell proliferation, and cell invasion and migration in vitro. LINC00886 physically interacts with PKR, and downregulated LINC00886 increases the elF2a activity to maintain cell survival by destabilizing PKR in ATC cell. Our findings identify LINC00886 as a novel biomarker in ATC, and suggest that the LINC00886-PKR-eIF2a signaling axis is a potential therapeutic target.

\section{Abbreviations}

ATC: anaplastic thyroid cancer; PDTC: poorly differentiated thyroid cancer; IncRNA: long non-cdoing RNA; DDTC: dedifferentiation thyroid cancer; FUSCC: Fudan University Shanghai Cancer Center; GEO: Gene Expression Omnibus; NT: normal thyroid; PTC: papillary thyroid cancer; PKR: protein kinase R; elF2a: eukaryotic initiation factor 2a; TCGA: The Cancer Genome Atlas; CPAT: Coding Potential Assessment Tool; CPC: Coding Potential Calculator; RACE: rapid amplification of the CDNA ends; siRNA: small interfering RNA; NC: negative control; ASO: anti-sense oligonucleotides; qPCR: quantitative real-time PCR; CCK-8: Cell Counting Kit-8; GSEA: Gene Set Enrichment Analysis; NES: normalized enrichment score; FDR: false discovery rate; RIP: RNA immunoprecipitation; SEM: standard error of the mean; SD: standard deviation; TDS: thyroid differentiation score; ROC: receiver operating characteristic; AUC: area under curve; OS: overall survival.

\section{Declarations}

\section{Ethics approval and consent to participate}

Our study was approved by the Medical Ethics Committee of the FUSCC, and all patients provided written informed consents for their specimens and information to be used for research and stored in the hospital database. All procedures performed in this study were in accordance with the 1964 Helsinki declaration and its later amendments or comparable ethical standards.

\section{Consent for publication}


Not applicable.

\section{Availability of data and materials}

The data generated or analysed during this study are included in this article and its supplementary information files.

\section{Competing interests}

The authors declare that they have no competing interests

\section{Funding}

The study was supported by the National Natural Science Foundation of China (81772854 to Qinghai Ji), the Science and Technology Commission of Shanghai Municipality (19411966600 to Yu Wang and 19ZR1410900 to Wenjun Wei) and the Shanghai Anticancer Association (SACA-AX106 to Yu Wang and SACA-CY19B01 to Ben Ma).

\section{Authors' contributions}

Yu Wang, Qinghai Ji, Wenjun Wei and Ben Ma designed the study. Ben Ma, Yi Luo and Weibo Xu were responsible for performing experiments. Tian Liao and Litao Han helped to guide some in vitro experiments. Ben Ma and Hongyi Jiang performed data analyses. Wanlin liu, Xiao Wang and Shishuai Wen contributed to collection of surgical samples and clinical information and data preparation. Ben Ma wrote the manuscript. Yu Wang, Qinghai Ji and Wenjun Wei revised the paper. All authors read and approved the final manuscript.

\section{Acknowledgments}

We thank Zhe Li and Shenglin Huang for their help in the present study. We thank The UCSC Xena Platform for cancer genomics data sets, the cBioPortal for Cancer Genomics and the GEO platform of NCBI.

\section{References}

1. Molinaro E, Romei C, Biagini A, Sabini E, Agate L, Mazzeo S, et al. Anaplastic thyroid carcinoma: from clinicopathology to genetics and advanced therapies. Nat Rev Endocrinol. 2017;13(11):644-60.

2. Huang NS, Shi X, Lei BW, Wei WJ, Lu ZW, Yu PC, et al. An Update of the Appropriate Treatment Strategies in Anaplastic Thyroid Cancer: A Population-Based Study of 735 Patients. Int J Endocrinol. 2019;2019(8428547).

3. Lim H, Devesa SS, Sosa JA, Check D, Kitahara CM. Trends in Thyroid Cancer Incidence and Mortality in the United States, 1974-2013. JAMA. 2017;317(13):1338-48. 
4. Ibrahimpasic T, Xu B, Landa I, Dogan S, Middha S, Seshan V, et al. Genomic Alterations in Fatal Forms of Non-Anaplastic Thyroid Cancer: Identification of MED12 and RBM10 as Novel Thyroid Cancer Genes Associated with Tumor Virulence. Clin Cancer Res. 2017;23(19):5970-80.

5. Landa I, Ibrahimpasic T, Boucai L, Sinha R, Knauf JA, Shah RH, et al. Genomic and transcriptomic hallmarks of poorly differentiated and anaplastic thyroid cancers. J Clin Invest. 2016;126(3):105266.

6. Ma B, Xu W, Wei W, Wen D, Lu Z, Yang S, et al. Clinicopathological and Survival Outcomes of WellDifferentiated Thyroid Carcinoma Undergoing Dedifferentiation: A Retrospective Study from FUSCC. Int J Endocrinol. 2018;2018(2383715.

7. Pozdeyev N, Gay LM, Sokol ES, Hartmaier R, Deaver KE, Davis S, et al. Genetic Analysis of 779 Advanced Differentiated and Anaplastic Thyroid Cancers. Clin Cancer Res. 2018;24(13):3059-68.

8. Ali MM, Akhade VS, Kosalai ST, Subhash S, Statello L, Meryet-Figuiere M, et al. PAN-cancer analysis of S-phase enriched IncRNAs identifies oncogenic drivers and biomarkers. Nat Commun. 2018;9(1):883.

9. Schmitt AM, Chang HY. Long Noncoding RNAs in Cancer Pathways. Cancer Cell. 2016;29(4):452-63.

10. Xu S, Kong D, Chen Q, Ping Y, Pang D. Oncogenic long noncoding RNA landscape in breast cancer. Mol Cancer. 2017;16(1):129.

11. Engreitz JM, Pandya-Jones A, McDonel P, Shishkin A, Sirokman K, Surka C, et al. The Xist IncRNA exploits three-dimensional genome architecture to spread across the $\mathrm{X}$ chromosome. Science. 2013;341(6147):1237973.

12. Gupta RA, Shah N, Wang KC, Kim J, Horlings HM, Wong DJ, et al. Long non-coding RNA HOTAIR reprograms chromatin state to promote cancer metastasis. Nature. 2010;464(7291):1071-6.

13. Hirose T, Virnicchi G, Tanigawa A, Naganuma T, Li R, Kimura H, et al. NEAT1 long noncoding RNA regulates transcription via protein sequestration within subnuclear bodies. Mol Biol Cell. 2014;25(1):169-83.

14. Kino T, Hurt DE, Ichijo T, Nader N, Chrousos GP. Noncoding RNA gas 5 is a growth arrest- and starvation-associated repressor of the glucocorticoid receptor. Sci Signal. 2010;3(107):a8.

15. Kretz M, Siprashvili Z, Chu C, Webster DE, Zehnder A, Qu K, et al. Control of somatic tissue differentiation by the long non-coding RNA TINCR. Nature. 2013;493(7431):231-5.

16. Wang $Y, X u Z$, Jiang J, Xu C, Kang J, Xiao L, et al. Endogenous miRNA sponge lincRNA-RoR regulates Oct4, Nanog, and Sox2 in human embryonic stem cell self-renewal. Dev Cell. 2013;25(1):69-80.

17. Thomson DW, Dinger ME. Endogenous microRNA sponges: evidence and controversy. Nat Rev Genet. 2016;17(5):272-83.

18. Prensner JR, Chinnaiyan AM. The emergence of IncRNAs in cancer biology. Cancer Discov. 2011;1(5):391-407.

19. Serghiou S, Kyriakopoulou A, loannidis JP. Long noncoding RNAs as novel predictors of survival in human cancer: a systematic review and meta-analysis. Mol Cancer. 2016;15(1):50. 
20. Evans JR, Feng FY, Chinnaiyan AM. The bright side of dark matter: IncRNAs in cancer. J Clin Invest. 2016;126(8):2775-82.

21. Arun G, Diermeier SD, Spector DL. Therapeutic Targeting of Long Non-Coding RNAs in Cancer. Trends Mol Med. 2018;24(3):257-77.

22. Ma B, Jiang H, Wen D, Hu J, Han L, Liu W, et al. Transcriptome Analyses Identify a Metabolic Gene Signature Indicative of Dedifferentiation of Papillary Thyroid Cancer. J Clin Endocrinol Metab. 2019;104(9):3713-25.

23. Tomas G, Tarabichi M, Gacquer D, Hebrant A, Dom G, Dumont JE, et al. A general method to derive robust organ-specific gene expression-based differentiation indices: application to thyroid cancer diagnostic. Oncogene. 2012;31(41):4490-8.

24. Dom G, Tarabichi M, Unger K, Thomas G, Oczko-Wojciechowska M, Bogdanova T, et al. A gene expression signature distinguishes normal tissues of sporadic and radiation-induced papillary thyroid carcinomas. Br J Cancer. 2012;107(6):994-1000.

25. Pita JM, Banito A, Cavaco BM, Leite V. Gene expression profiling associated with the progression to poorly differentiated thyroid carcinomas. Br J Cancer. 2009;101(10):1782-91.

26. von Roemeling CA, Marlow LA, Pinkerton AB, Crist A, Miller J, Tun HW, et al. Aberrant lipid metabolism in anaplastic thyroid carcinoma reveals stearoyl CoA desaturase 1 as a novel therapeutic target. J Clin Endocrinol Metab. 2015;100(5):E697-709.

27. Barrett T, Wilhite SE, Ledoux P, Evangelista C, Kim IF, Tomashevsky M, et al. NCBI GEO: archive for functional genomics data sets-update. Nucleic Acids Res. 2013;41(Database issue):D991-5.

28. Edgar R, Domrachev M, Lash AE. Gene Expression Omnibus: NCBI gene expression and hybridization array data repository. Nucleic Acids Res. 2002;30(1):207-10.

29. Irizarry RA, Hobbs B, Collin F, Beazer-Barclay YD, Antonellis KJ, Scherf U, et al. Exploration, normalization, and summaries of high density oligonucleotide array probe level data. Biostatistics. 2003;4(2):249-64.

30. Subramanian A, Tamayo P, Mootha VK, Mukherjee S, Ebert BL, Gillette MA, et al. Gene set enrichment analysis: a knowledge-based approach for interpreting genome-wide expression profiles. Proc Natl Acad Sci U S A. 2005;102(43):15545-50.

31. Murugan AK, Munirajan AK, Alzahrani AS. Long noncoding RNAs: emerging players in thyroid cancer pathogenesis. Endocr Relat Cancer. 2018;25(2):R59-82.

32. Mahmoudian-Sani MR, Jalali A, Jamshidi M, Moridi H, Alghasi A, Shojaeian A, et al. Long NonCoding RNAs in Thyroid Cancer: Implications for Pathogenesis, Diagnosis, and Therapy. Oncol Res Treat. 2019;42(3):136-42.

33. Sedaghati M, Kebebew E. Long noncoding RNAs in thyroid cancer. Curr Opin Endocrinol Diabetes Obes. 2019;26(5):275-81.

34. Sui F, Ji M, Hou P. Long non-coding RNAs in thyroid cancer: Biological functions and clinical significance. Mol Cell Endocrinol. 2018;469(11-22. 
35. Lan L, Cao H, Chi W, Meng W, Zhao L, Cui W, et al. Aberrant DNA hypermethylation-silenced LINC00886 gene accelerates malignant progression of laryngeal carcinoma. Pathol Res Pract. 2020;216(4):152877.

36. Ransohoff JD, Wei Y, Khavari PA. The functions and unique features of long intergenic non-coding RNA. Nat Rev Mol Cell Biol. 2018;19(3):143-57.

37. Guo C, Shao R, Correa AM, Behrens C, Johnson FM, Raso MG, et al. Prognostic significance of combinations of RNA-dependent protein kinase and EphA2 biomarkers for NSCLC. J Thorac Oncol. 2013;8(3):301-8.

38. Hii SI, Hardy L, Crough T, Payne EJ, Grimmett K, Gill D, et al. Loss of PKR activity in chronic lymphocytic leukemia. Int J Cancer. 2004;109(3):329-35.

39. Kwon HC, Moon CH, Kim SH, Choi HJ, Lee HS, Roh MS, et al. Expression of double-stranded RNAactivated protein kinase (PKR) and its prognostic significance in lymph node negative rectal cancer. Jpn J Clin Oncol. 2005;35(9):545-50.

40. Shimada A, Shiota G, Miyata H, Kamahora T, Kawasaki H, Shiraki K, et al. Aberrant expression of double-stranded RNA-dependent protein kinase in hepatocytes of chronic hepatitis and differentiated hepatocellular carcinoma. Cancer Res. 1998;58(19):4434-8.

41. Garcia-Ortega MB, Lopez GJ, Jimenez G, Garcia-Garcia JA, Conde V, Boulaiz H, et al. Clinical and therapeutic potential of protein kinase PKR in cancer and metabolism. Expert Rev Mol Med. 2017;19(e9.

42. Lee SB, Esteban M. The interferon-induced double-stranded RNA-activated protein kinase induces apoptosis. Virology. 1994;199(2):491-6.

43. Yoon CH, Lee ES, Lim DS, Bae YS. PKR, a p53 target gene, plays a crucial role in the tumorsuppressor function of p53. Proc Natl Acad Sci U S A. 2009;106(19):7852-7.

44. Mounir Z, Krishnamoorthy JL, Robertson GP, Scheuner D, Kaufman RJ, Georgescu MM, et al. Tumor suppression by PTEN requires the activation of the PKR-elF2alpha phosphorylation pathway. Sci Signal. 2009;2(102):a85.

45. Roh MS, Kwak JY, Kim SJ, Lee HW, Kwon HC, Hwang TH, et al. Expression of double-stranded RNAactivated protein kinase in small-size peripheral adenocarcinoma of the lung. Pathol Int. 2005;55(11):688-93.

46. Ding J, Zhao J, Huan L, Liu Y, Qiao Y, Wang Z, et al. Inflammation-induced LINC00665 increases the malignancy through activating PKR/NF-kappaB pathway in hepatocellular carcinoma. Hepatology. 2020.

\section{Figures}




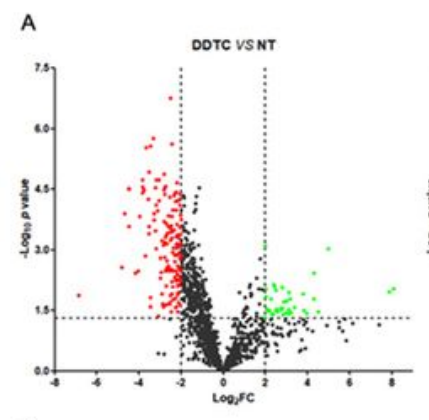

C

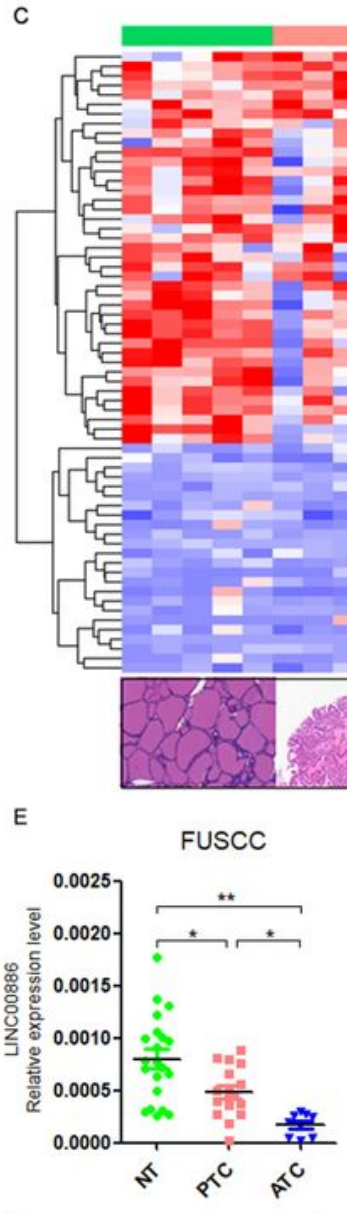

H

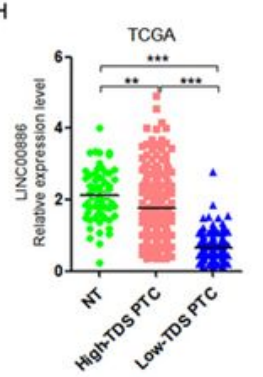

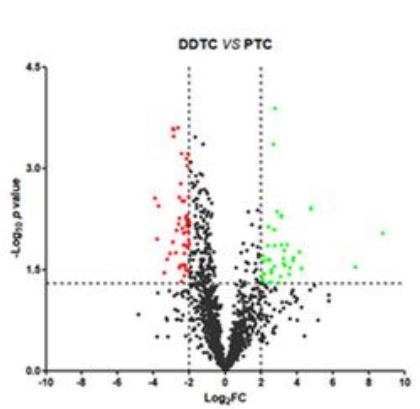

D
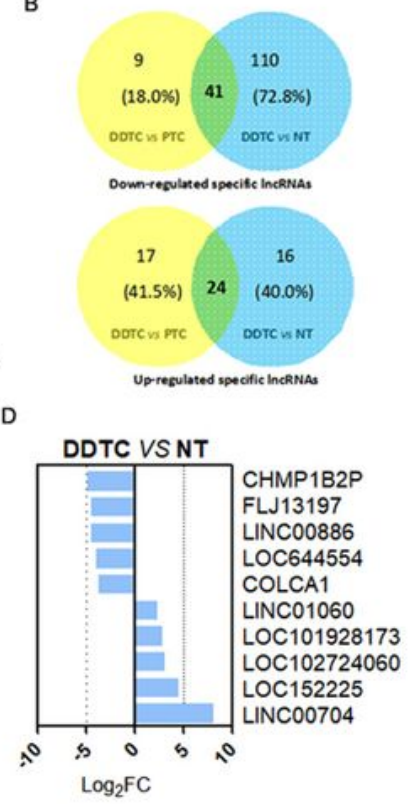

DDTC VS PTC
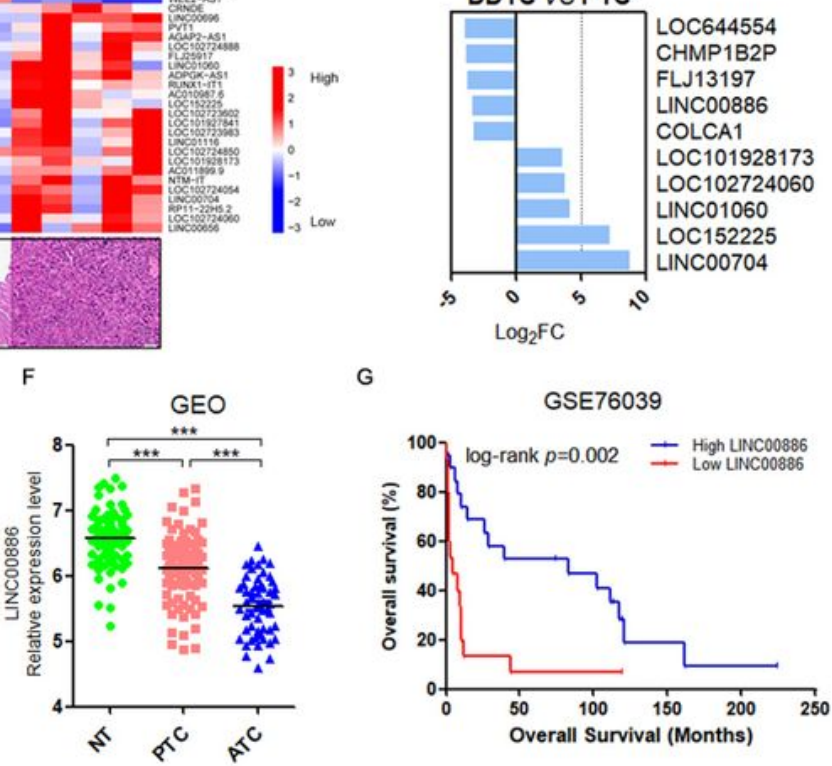

\begin{tabular}{|l|c|c|}
\hline Risk factors & OR $(95 \% \mathrm{CI})$ & P value \\
\hline ETE & $0.468(0.344-0.636)$ & $<0.001$ \\
\hline LNM & $0.424(0.322-0.558)$ & $<0.001$ \\
\hline T3/T4 stage & $0.618(0.487-0.785)$ & $<0.001$ \\
\hline II/III/IV stage & $0.722(0.542-0.960)$ & 0.025 \\
\hline
\end{tabular}

Figure 1

LINC00886 is indicative of thyroid cancer dedifferentiation and significantly suppressed in ATC. (A) Volcano maps showing the deregulated IncRNAs in DDTCs compared with PTCs and NTs, log2(fold change) ( $F C) \geq 2, p<0.05$; (B) Venn maps exhibiting the overlapping 65 IncRNAs deregulated in DDTCs compared with PTCs and NTs; (C) The heatmap of the 65 differential IncRNAs' distribution in DDTCs, PTCs and NTs; (D) Differential FC of top ten IncRNAs calculated by log2FC in DDTCs compared with 
PTCs and NTs; (E) Comparison of LINC00886 expression by quantitative real-time polymerase chain reaction (qPCR) among ATCs, PTCs and NTs from FUSCC; (F) Comparison of LINC00886 expression among ATCs, PTCs and NTs from the combined GEO cohort; (G) The Kaplan-Meier method was used to construct overall survival (OS) curves of LINC00886 expression in thyroid cancer patients using the GSE76039 cohort; $(H)$ The PTC patients from the TCGA cohort were divided into a low-differentiated group and a high-differentiated group based on the median value of thyroid differentiation score (TDS), comparison of LINC00886 expression was conducted among the low-TDS group, the high-TDS group and NTs; (I) Pearson's correlation was performed to analyse the correlation of LINC00886 with the TDS in the TCGA cohort; $(J)$ Univariate logistic regression analyses were performed to determine associations of LINC00886 with clinicopathological factors in the TCGA cohort. Values are expressed as the mean \pm SEM, ${ }^{*} \mathrm{p}<0.05,{ }^{*} \mathrm{p}<0.01, * * * \mathrm{p}<0.001$.
A
B Histological risk

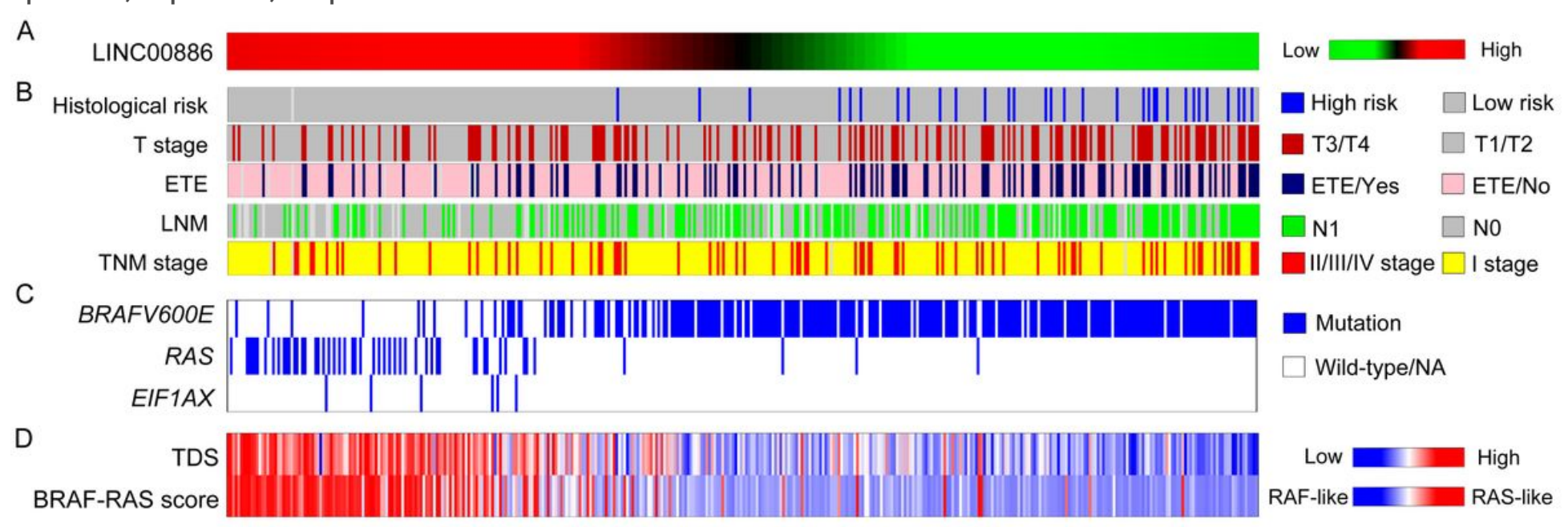

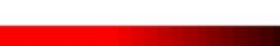

\section{Figure 2}

Significant associations of LINC00886 expression with clinicopathological parameters and genetic mutations. PTC patients of the TCGA cohort were ranked by LINC00886 (A), clinicopathological factors (B), genetic mutations (C), TDS and BRAF-RAS score (D). 
A

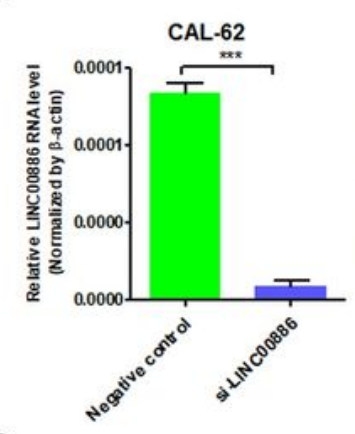

D

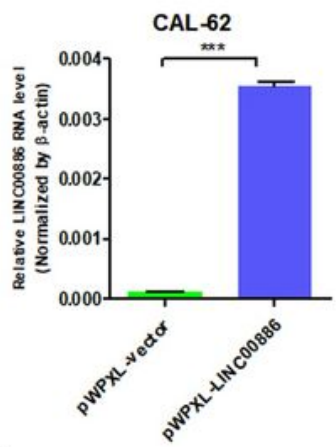

G

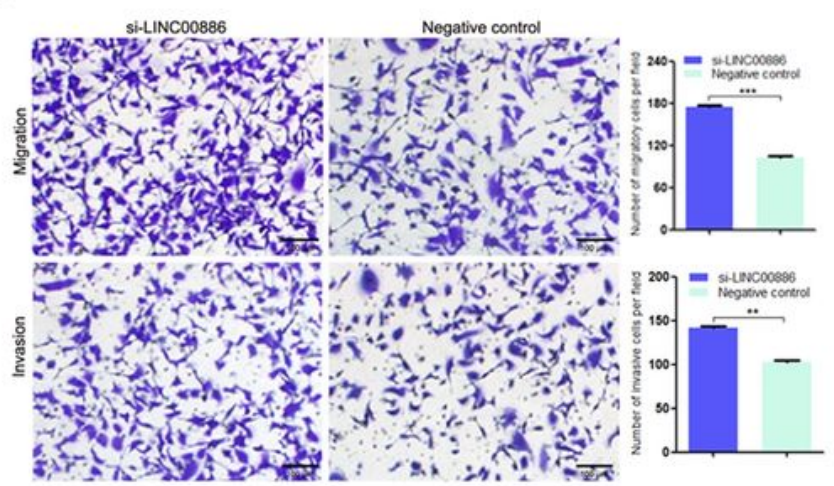

C

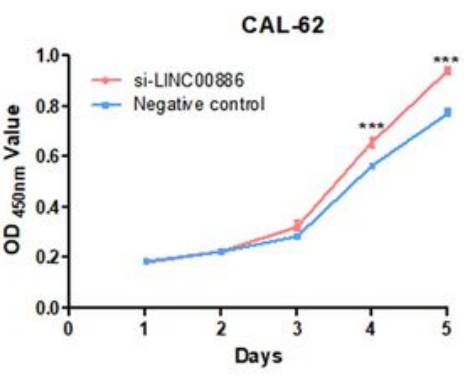

E
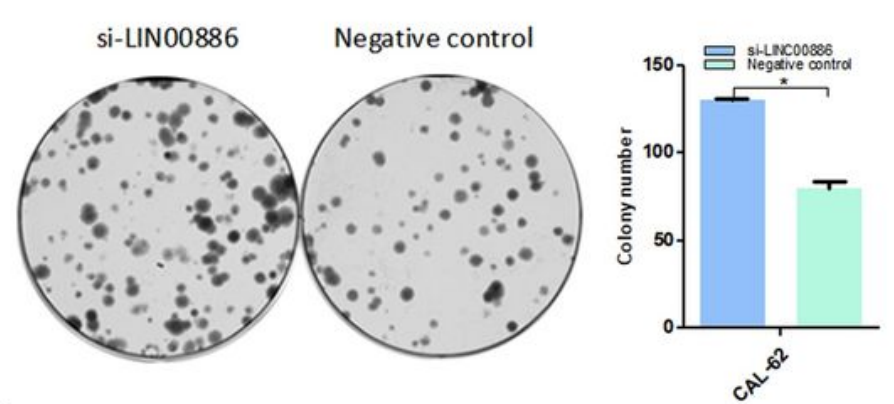

CAL 62
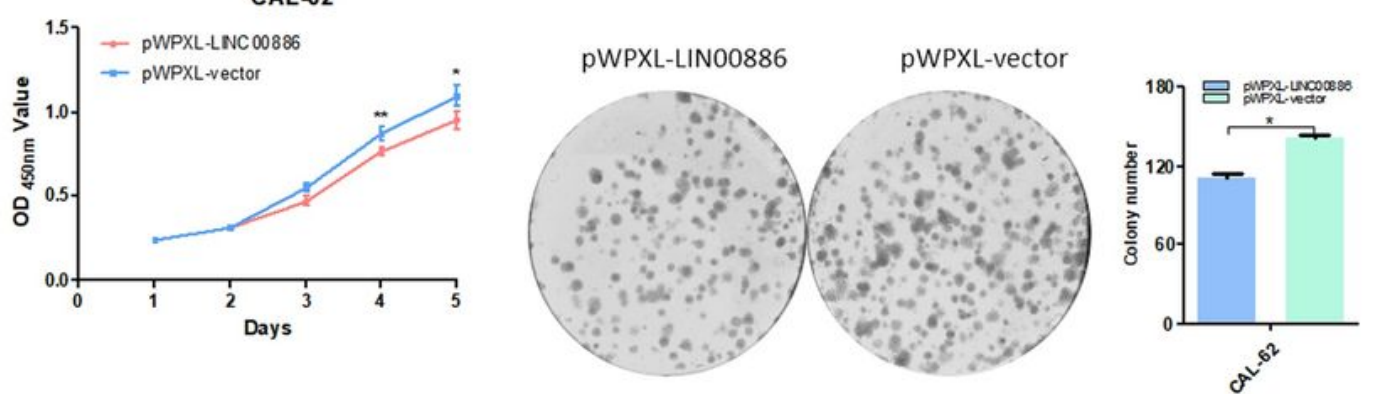

H

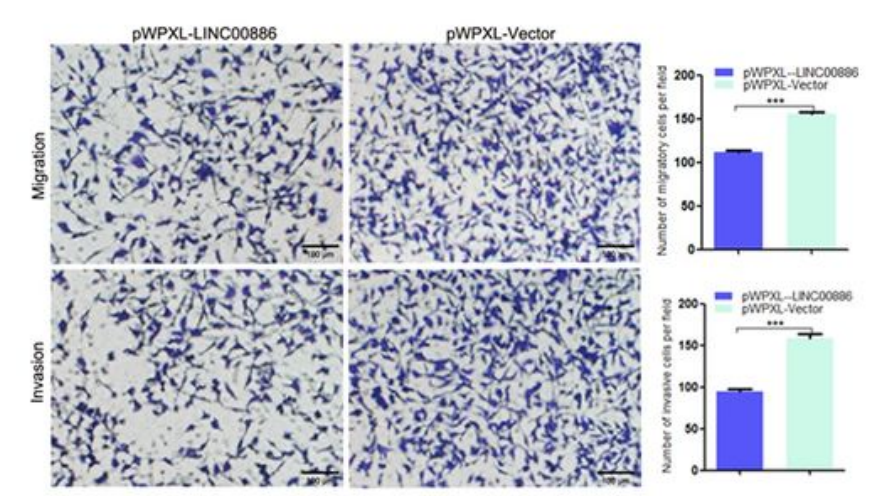

K

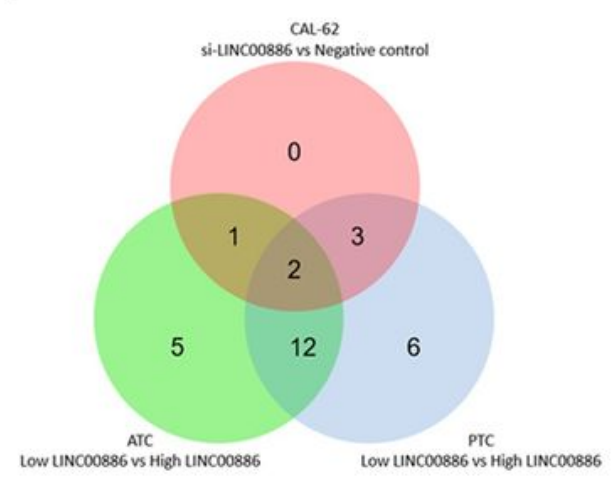

J
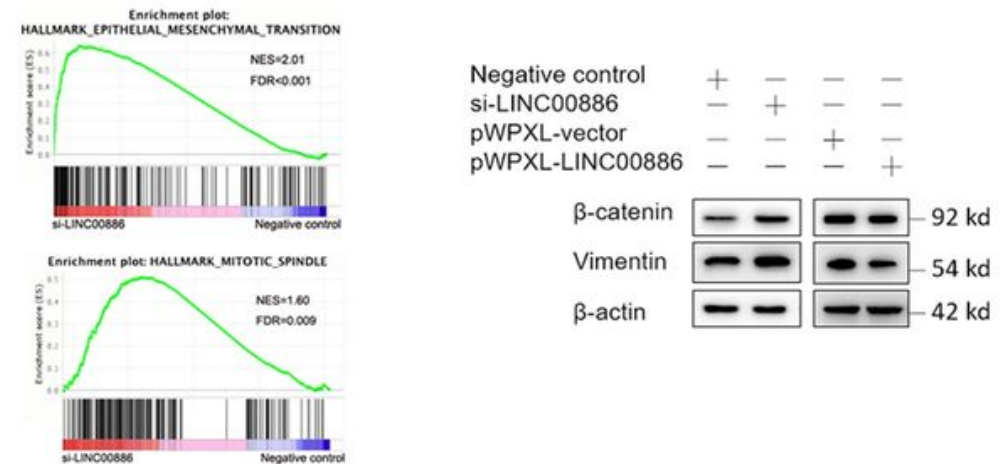

Figure 3

LINC00886 negatively regulates ATC cell proliferation and migration and invasion. (A) Analysis of LINC00886 knockdown by LINC00886 smart silencer in CAL-62 using quantitative real-time polymerase chain reaction (qPCR); (B) Cell Counting Kit-8 (CCK-8) assays of LINC00886 knockdown in CAL-62; (C) Cell colony formation assays of LINC00886 knockdown in CAL-62; (D) Analysis of overexpression of LINC00886 by transfecting the pWPXL-LINC00886 plasmids in CAL-62 using qPCR; (E) CCK-8 assays of 
LINC00886 overexpression in CAL-62; (F) Cell colony formation assays of LINC00886overexpression in CAL-62; (G) Cell migration and invasion assays of LINC00886 knockdown in CAL-62; $(\mathrm{H})$ Cell migration and invasion assays of LINC00886 overexpression in CAL-62; (I) Overlapped GSEA analyses using RNA sequencing data of CAL-62 cells of LINC00886 knockdown and negative control as well as transcriptome data of the high-LINC00886 and low-LINC00886 expression groups from the GEO ATC cohort and the TCGA PTC cohort; (J) Two overlapped signaling pathways including epithelial-mesenchymal transition and mitotic spindle enriched in LINC00886-downregulation group; $(K) \beta$-catenin and vimentin expression of LINC00886 knockdown and overexpression by western blot. Values are expressed as the mean \pm SEM, $\mathrm{n}=3$ in $(\mathrm{A})-(\mathrm{H}),{ }^{*} \mathrm{p}<0.05, * * \mathrm{p}<0.01, * * * \mathrm{p}<0.001$.

A

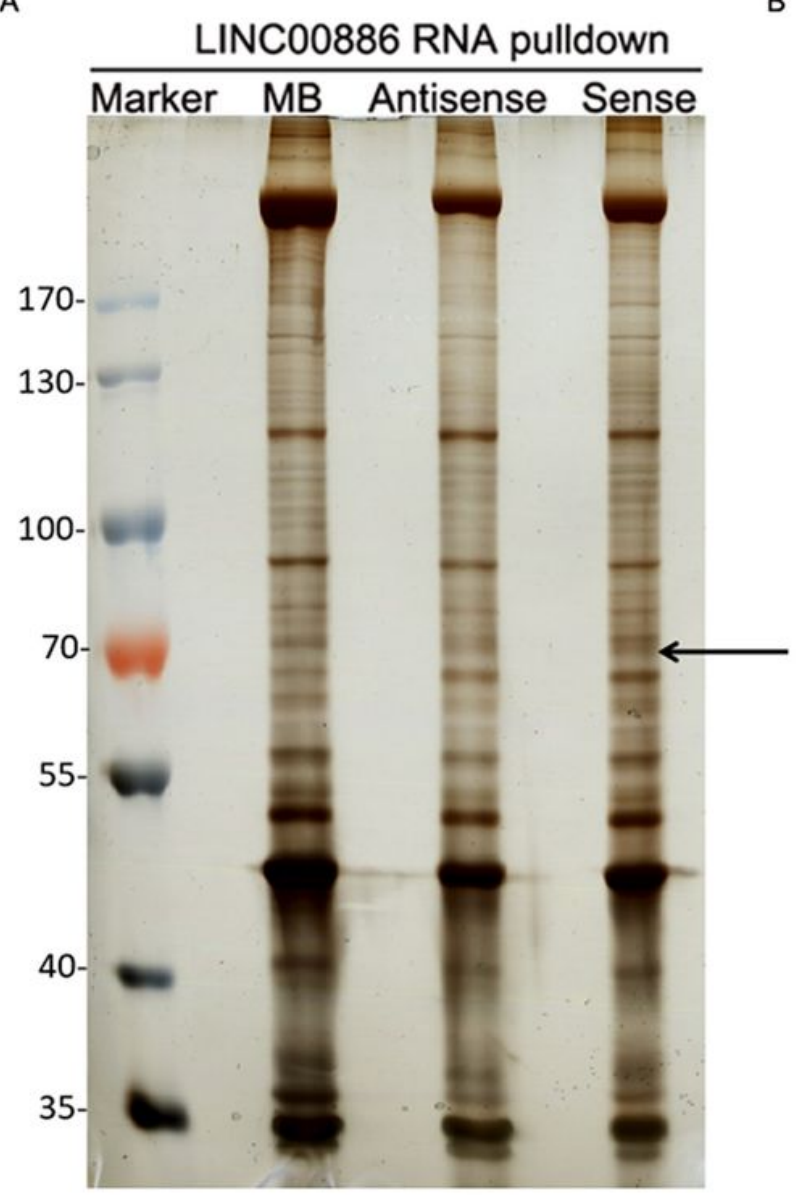

B

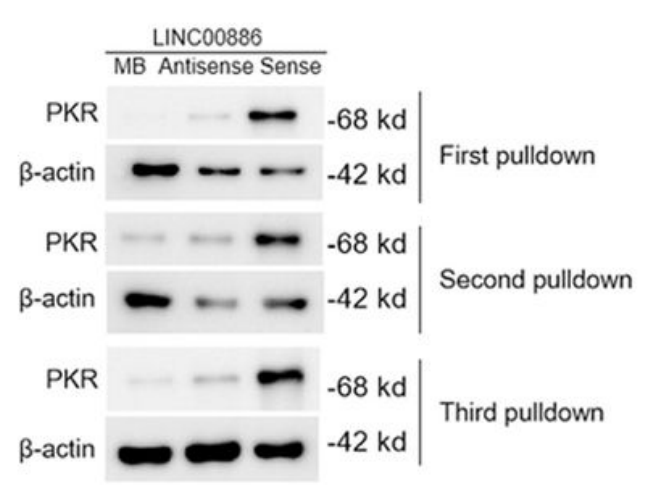

C

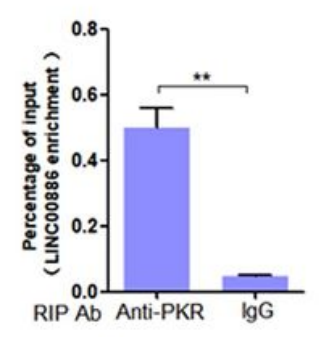

D

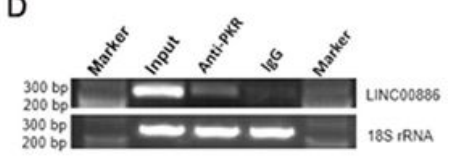

$\mathrm{E}$

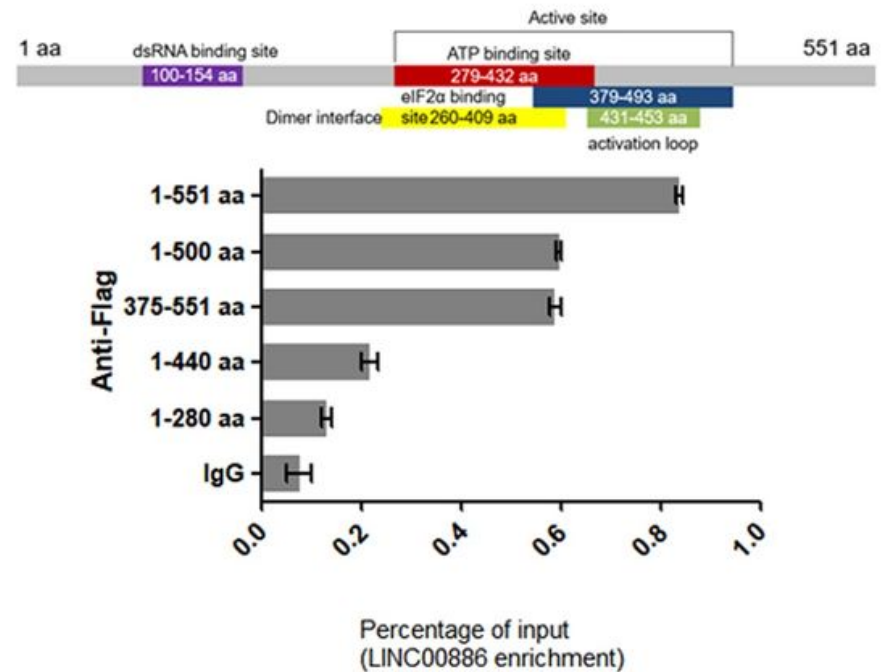

Figure 4

LINC00886 targets PKR in ATC cell. (A) Specific protein bands around 70 kd visualized by silver staining; (B) PKR was identified by mass spectrometry analysis and confirmed by western blot; (C) Relative levels of LINC00886 immunoprecipitated with PKR or IgG antibody analyzed by quantitative real-time polymerase chain reaction (qPCR); (D) Real-time polymerase chain reaction (RT-PCR) assays used to detect LINC00886; (E) Deletion mapping for the domains of PKR that binds to LINC00886. RIP analysis for LINC00886 enrichment in cells transiently transfected with plasmids containing the indicated FLAGtagged full-length or truncated constructs. Values are expressed as the mean $\pm S E M, n=3$ in (C), ${ }^{\star} p<0.05$, $\star \star x p 0.01, * \star * p<0.001$. 
A

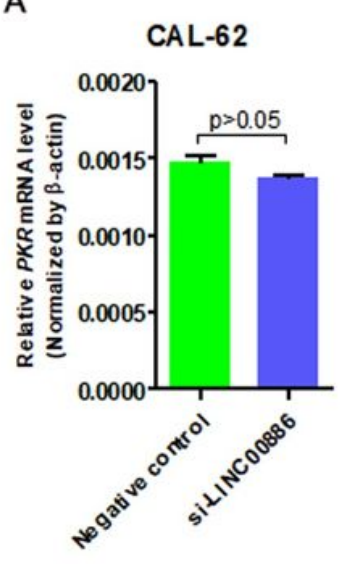

D

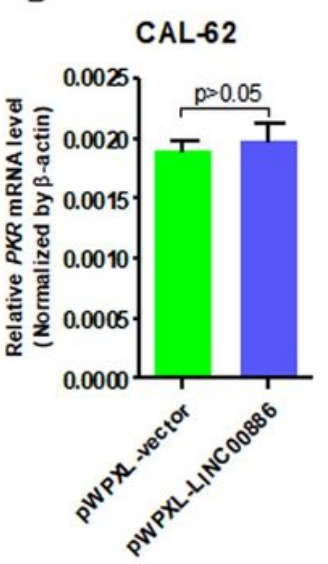

$\mathrm{CHX}(50 \mu \mathrm{g} / \mathrm{ml})$

$0 \mathrm{~h} \quad 6 \mathrm{~h} 12 \mathrm{~h} 18 \mathrm{~h} 24 \mathrm{~h}$

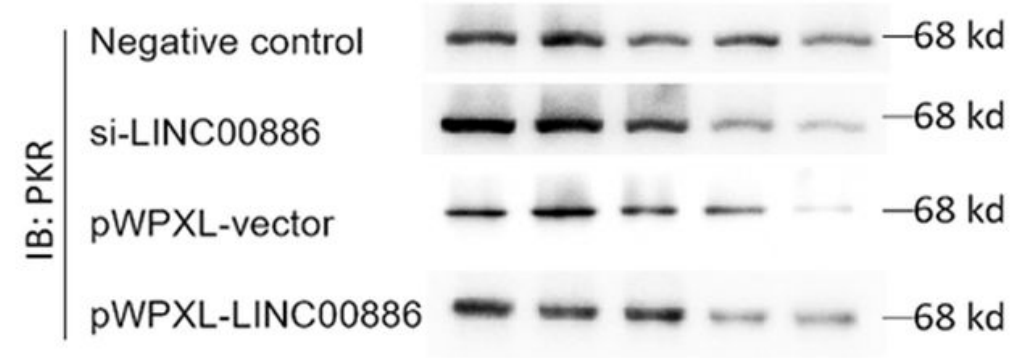

F

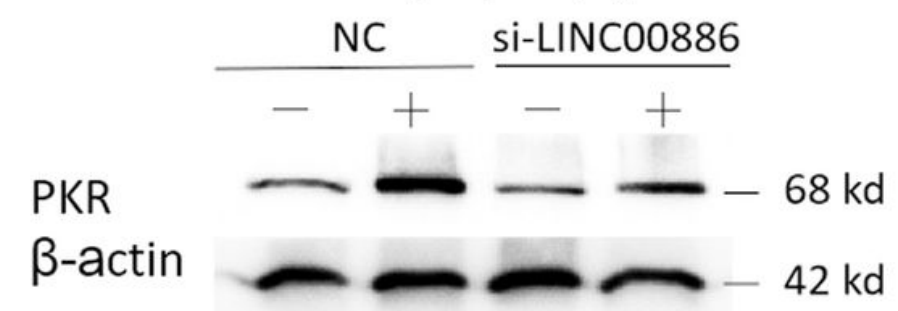

Negative control si-LINC00886 pWPXL-vector pWPXL-LINC00886

PKR

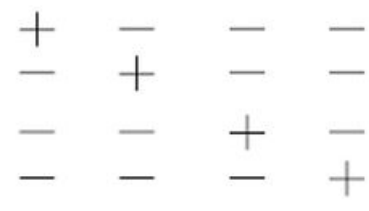

$\beta$-actin

$-68 \mathrm{kd}$ $-42 \mathrm{kd}$

E

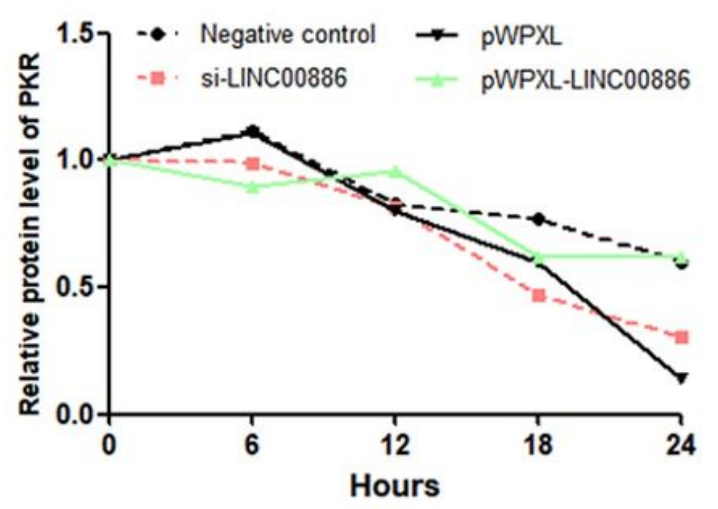

G

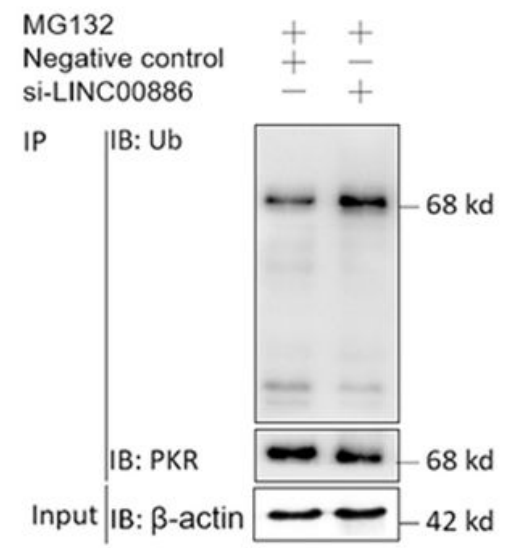

\section{Figure 5}

LINC00886 affects PKR stability in ATC cell. (A) Relative levels of PKR mRNA by quantitative real-time polymerase chain reaction (qPCR) in LINC00886-knockdown CAL-62 cells; (B) Relative levels of PKR mRNA by qPCR in LINC00886-overexpressed CAL-62 cells; (C) PKR protein levels by western blot in LINC00886-knockdown and LINC00886-overexpressed CAL-62 cells; (D-E) The immunoblotting and densitometry analyses for the PKR levels in LINC00886-knockdown cells, LINC00886-overexpressed cells, or the control cells treated with cycloheximide $(\mathrm{CHX}, 50 \mu \mathrm{g} / \mathrm{ml})$ for the indicated times, the relative fold of the level at $0 \mathrm{~h}$; (F) LINC00886-knockdown cells were treated with MG132 (25 $\mu \mathrm{M})$ for $12 \mathrm{~h}$. Immunoblotting for PKR protein levels in the indicated cells; (G) The lysates of LINC00886-knockdown 
cells were immunoprecipitated (IP) with anti-PKR antibody and immunoblotted with the ubiquitin-specific antibody. PKR and $\beta$-actin served as the loading control.

A

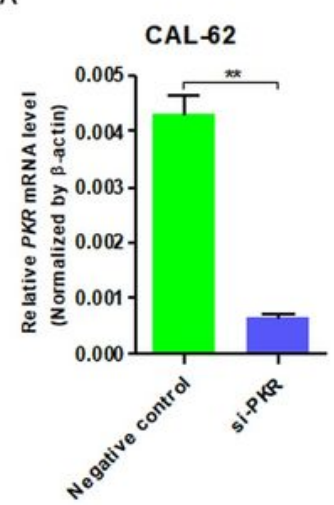

D

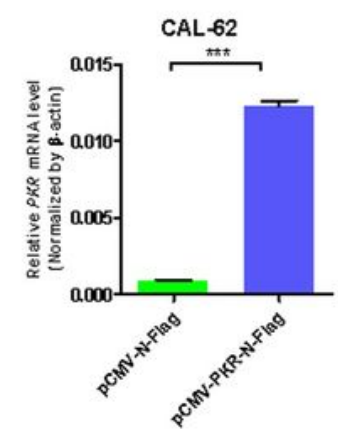

G

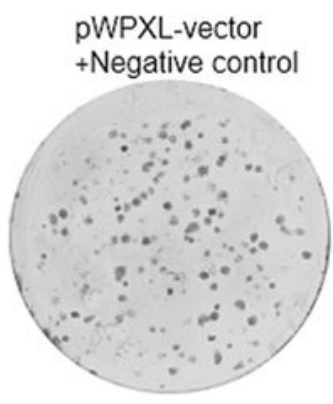

H
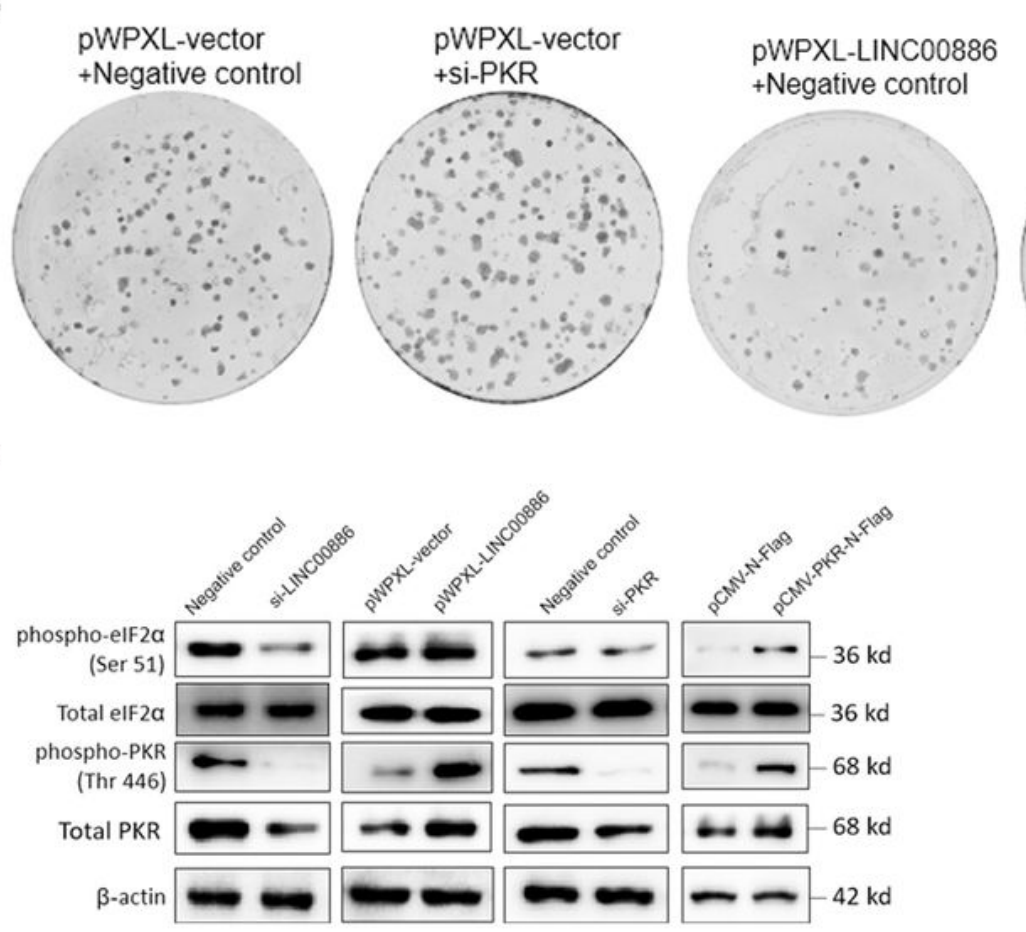

si-PKR Negative control
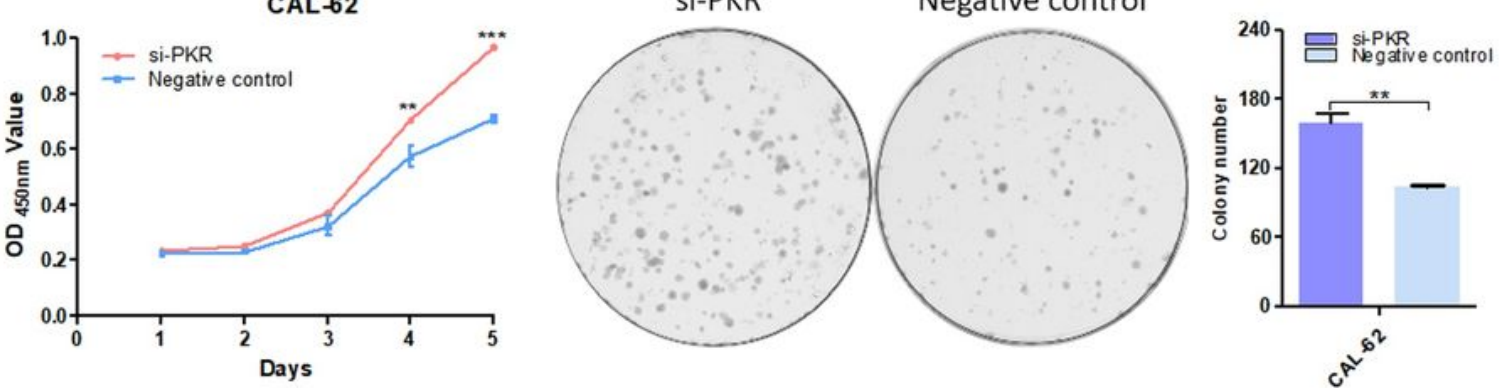

$E$

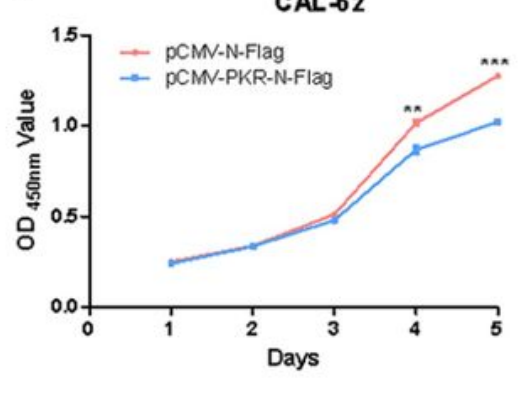

$\mathrm{F}$
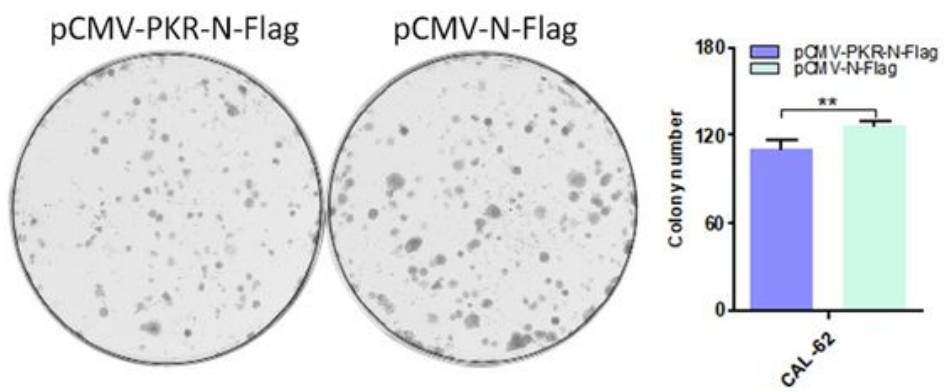
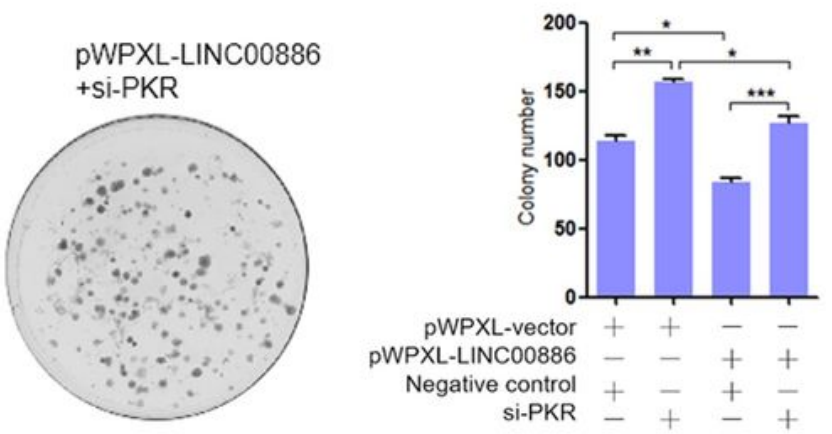

1

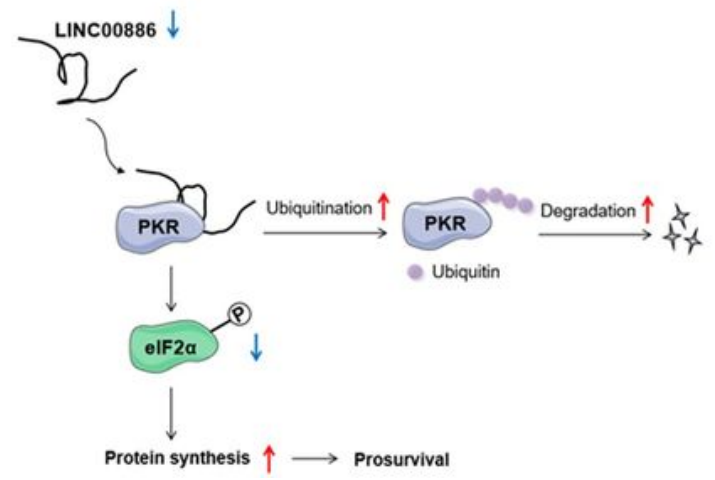

Figure 6

The PKR/elF2a signaling axis mediates functional regulation of LINC00886. (A) Analysis of PKR knockdown by siRNA in CAL-62 using quantitative real-time polymerase chain reaction (qPCR); (B) Cell Counting Kit-8 (CCK-8) assays of PKR knockdown in CAL-62; (C) Cell colony formation assays of PKR 
knockdown in CAL-62; (D) Analysis of overexpression of PKR by transfecting the pCMV-PKR-N-Flag plasmids in CAL-62 using qPCR; (E) CCK-8 assays of PKR overexpression in CAL-62; (F) Cell colony formation assays of LINC00886 overexpression in CAL-62; $(G)$ Rescue assays of colony formation assay were performed after silencing PKR in pWPXL-LINC00886 CAL-62 cells; $(\mathrm{H})$ Immunoblotting analysis of total PKR, phosphorylated PKR (Thr446), total elF2 $a$ and phosphorylated elF2a (Ser51) in LINC00886knockdown, LINC00886-overexpressed, PKR-knockdown and PKR-overexpressed CAL-62 cells. $\beta$-actin served as the internal control in (A), (D) and (H); (I) Integrated model depicting IncRNA LINC00886 as a suppressor in anaplastic thyroid cancer. The working model shows that LINC00886 downregulation destabilizing PKR through directly interacting with PKR in a ubiquitin/proteasome dependent manner, which enhanced elF $2 a$ activity to maintain cancer cell survival. Values are expressed as the mean $\pm S E M$, $n=3$ in $(A)-(G) ;{ }^{*}<00.05, * * p<0.01, * \star * p<0.001$.

\section{Supplementary Files}

This is a list of supplementary files associated with this preprint. Click to download.

- TableS7.doc

- TableS7.doc

- TableS6.doc

- TableS6.doc

- Tables5.doc

- TableS5.doc

- Tables4.doc

- TableS4.doc

- TableS3.doc

- Tables3.doc

- Tables2.pdf

- Tables2.pdf

- TableS1.doc

- TableS1.doc

- Fig.S2.tif

- Fig.S2.tif

- Fig.S3.tif

- Fig.S3.tif

- Fig.S1.tif

- Fig.S1.tif 